\title{
Doublet-Triplet Fermionic Dark Matter
}

\author{
Athanasios Dedes* And Dimitrios Karamitros ${ }^{\dagger}$ \\ Department of Physics, Division of Theoretical Physics, \\ University of Ioannina, GR 45110, Greece
}

June 24, 2015

\begin{abstract}
We extend the Standard Model (SM) by adding a pair of fermionic $S U(2)$-doublets with opposite hypercharge and a fermionic $S U(2)$-triplet with zero hypercharge. We impose a discrete $Z_{2}$-symmetry that distinguishes the SM fermions from the new ones. Then, gauge invariance allows for two renormalizable Yukawa couplings between the new fermions and the SM Higgs field, as well as for direct masses for the doublet $\left(M_{D}\right)$ and the triplet $\left(M_{T}\right)$. After electroweak symmetry breaking, this model contains, in addition to SM particles, two charged Dirac fermions and a set of three neutral Majorana fermions, the lightest of which contributes to Dark Matter (DM). We consider a case where the lightest neutral fermion is an equal admixture of the two doublets with mass $M_{D}$ close to the $Z$ boson mass. This state remains stable under radiative corrections thanks to a custodial $S U(2)$-symmetry and is consistent with the experimental data from oblique electroweak corrections. Moreover, the amplitudes relevant to spin-dependent or independent nucleusDM particle scattering cross section both vanish at tree level. They arise at one loop at a level that may be observed in near future DM direct detection experiments. For Yukawa couplings comparable to the top-quark, the DM particle relic abundance is consistent with observation, not relying on co-annihilation or resonant effects and has a mass at the electroweak scale. Furthermore, the heavier fermions decay to the DM particle and to electroweak gauge bosons making this model easily testable at the LHC. In the regime of interest, the charged fermions suppress the Higgs decays to diphoton by $45-75 \%$ relative to SM prediction.
\end{abstract}

\footnotetext{
*email: adedes@cc.uoi.gr
}

${ }^{\dagger}$ email: dkaramit@cc.uoi.gr 


\section{Introduction}

Motivated by astrophysical observations that suggest the existence of Dark Matter [1], we would like to propose a model with a fermionic Weakly Interacting Massive Particle (WIMP) $\left(\chi_{1}^{0}\right)$ whose mass and couplings are directly associated to electroweak scale providing the universe with the right thermal relic density abundance, not "tuned" by co-annihilation or resonance effects. Today, as opposed to five years ago, attempts of this sort immediately face difficulties due to strong experimental bounds $[2,3]^{1}$ from direct searches on nucleus recoiling energy in WIMP-nucleus scattering processes [5]. As a result, $Z$ - and Higgs- boson couplings to $\chi_{1}^{0}$-pairs are strongly constrained and usually come into conflict with values of couplings required from the observed [6] DM relic abundance. We therefore seek for a model at which, at least at tree level, these couplings vanish by a symmetry and at the same time the observed relic density is reproduced. We then discuss further consequences of this idea at Large Hadron Collider (LHC).

We consider a minimal model which realises this situation, hence, in addition to Standard Model (SM) particles, we add a pair of Weyl-fermion doublets $\overline{\mathbf{D}}_{\mathbf{1}} \sim\left(\mathbf{1}^{\mathrm{c}}, \mathbf{2}\right)_{-\mathbf{1}}$ and $\overline{\mathbf{D}}_{\mathbf{2}} \sim\left(\mathbf{1}^{\mathbf{c}}, \mathbf{2}\right)_{+\mathbf{1}}$ with opposite hypercharges, and a Weyl-fermion triplet $\mathbf{T} \sim\left(\mathbf{1}^{\mathbf{c}}, \mathbf{3}\right)_{\mathbf{0}}$ with zero hypercharge. The new Yukawa interactions allowed by gauge invariance and renormalizability are given by ${ }^{2}$

$$
\mathcal{L}_{\text {Yuk }} \supset Y_{1} \mathbf{T} \mathbf{H} \tau \overline{\mathbf{D}}_{\mathbf{1}}+Y_{2} \mathbf{T} \mathbf{H}^{\dagger} \tau \overline{\mathbf{D}}_{\mathbf{2}}-M_{D} \overline{\mathbf{D}}_{\mathbf{1}} \overline{\mathbf{D}}_{\mathbf{2}}-\frac{1}{2} M_{T} \mathbf{T} \mathbf{T},
$$

with $\tau$ being the Pauli matrices. A $Z_{2}$-discrete parity symmetry has been employed to guarantee that the new fermions interact always in pairs. Clearly, $\mathcal{L}_{\text {Yuk }}$ is invariant under the interchange symmetry $\mathbf{H} \leftrightarrow \mathbf{H}^{\dagger}$ and $\overline{\mathbf{D}}_{\mathbf{1}} \leftrightarrow \overline{\mathbf{D}}_{\mathbf{2}}$ when $Y_{1}=Y_{2} \equiv Y$. Then, it is very easy to see that in this limit, one eigenvalue with mass $M_{D}$, of the neutral $(3 \times 3)$ mixing mass matrix, decouples from the two heavier ones and the latter is degenerate with the two eigenvalues of the $(2 \times 2)$ charged fermion mass matrix. At tree level approximation, except for the lightest neutral fermion $\left(\chi_{1}^{0}\right)$, all other masses are controlled by the Yukawa coupling $Y$. The state with $m_{\chi_{1}^{0}}=M_{D}$ is our DM candidate particle. This particle state contains an equal admixture of the two doublets but has no triplet component,

$$
\left|\chi_{1}^{0}\right\rangle=0 \cdot|\mathbf{T}\rangle+\frac{1}{\sqrt{2}}\left|\overline{\mathbf{D}}_{\mathbf{1}}\right\rangle+\frac{1}{\sqrt{2}}\left|\overline{\mathbf{D}}_{\mathbf{2}}\right\rangle .
$$

Because the neutral component of the triplet does not participate in $\left|\chi_{1}^{0}\right\rangle$, the latter does not couple to the Higgs boson at tree level. It does not couple to the $Z$-gauge boson neither because of its equal admixture of neutral particles with opposite weak isospin. The situation here is analogous to the custodial symmetry [7 imposed in strongly coupled EW scenarios, where the "custodian" new particles are inserted in a similar way to protect certain quarkgauge boson couplings to obtain large radiative corrections 8 -11].

The couplings $h \chi_{1}^{0} \chi_{1}^{0}$ and $Z \chi_{1}^{0} \chi_{1}^{0}$ vanish at tree level, and as a result there are no $s$-channel amplitudes contributing to the annihilation cross section. However, there are off-diagonal interactions such as e.g., $Z \chi_{1}^{0} \chi_{2}^{0}$ that render the $t, u$-channel amplitudes non-zero but yet

\footnotetext{
${ }^{1}$ There are of course tantalising hints from DAMA, CoGeNT, CRESST-II and CDMS-Si experiments but these face stringent constraints from recent null result experiments like XENON100 and LUX making puzzling any theoretical interpretation of them all. For a recent review, see ref. 4].

${ }^{2}$ All gauge group indices are suppressed in this equation. Its detailed form is given below in eq. 2.9 .
} 


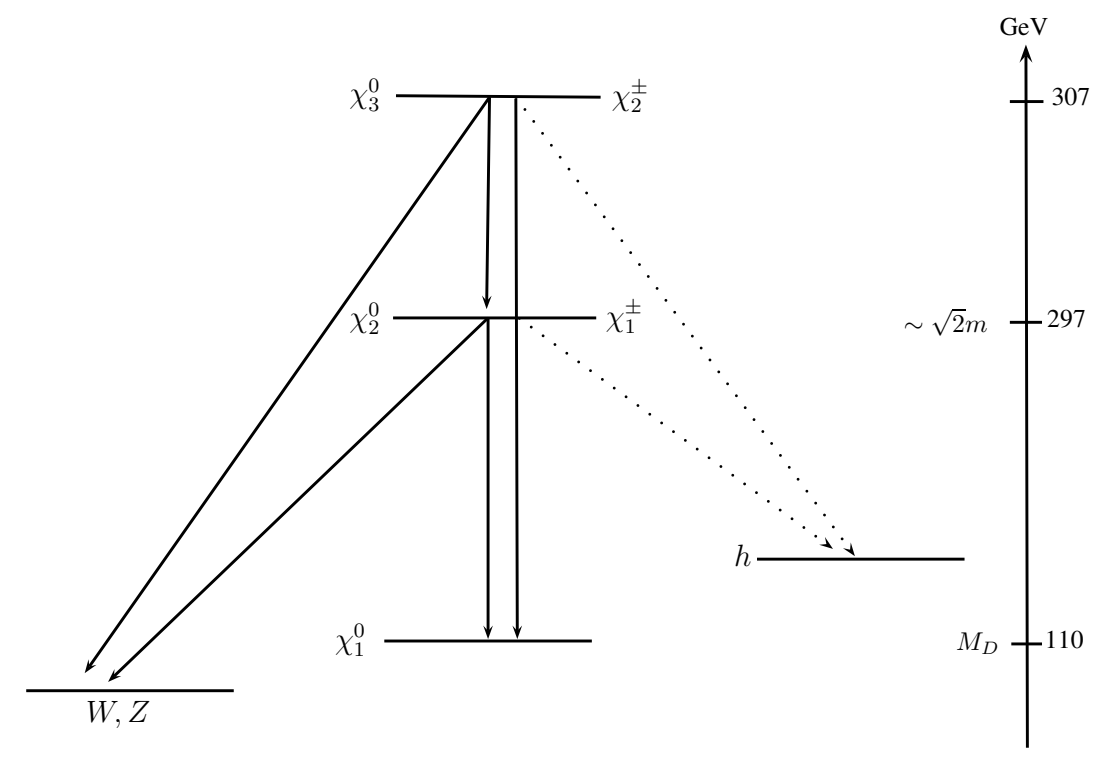

Figure 1: A sketch for the mass spectrum and decays of the new physical doublet and triplet fermions. The lightest neutral particle, $\chi_{1}^{0}$ is an equal admixture of the two doublets and has mass $M_{D}$. Particles $\chi_{2}^{0}\left(\chi_{3}^{0}\right)$ and $\chi_{1}^{ \pm}\left(\chi_{2}^{ \pm}\right)$are mass degenerate. For the spectrum masses written to the right we have chosen $M_{D}=110 \mathrm{GeV}, M_{T}=100 \mathrm{GeV}$ and $m=Y v=200$ $\mathrm{GeV}$. It provides the correct relic density abundance for dark matter [see Section 4] and is currently about $\sim 10$ times less sensitive to current direct detection searches [see Section 5].

suppressed enough to obtain the right relic density $\Omega_{\chi}$ for $M_{D} \approx 100 \mathrm{GeV}$ and $Y \approx 1$. Roughly speaking, the spectrum of the model where this happens is shown schematically in Fig. 1. Typically, the lightest stable new particle $\left(m_{\chi_{1}^{0}} \approx 110 \mathrm{GeV}\right)$ is in the vicinity of the EW scale while all other neutral and charged fermions are above $m \equiv Y v$ which is taken around the top quark mass. The splitting of the charged fermions is also controlled by the triplet mass $\left(M_{T}\right)$. Therefore, the parameters of the model are just three: $M_{D}, M_{T}$ and $m$.

Naively, one may think that this model is similar to the "wino-higgsino" sector of the MSSM [12] or it is an extended variant of the singlet-doublet DM model of refs. [13 16]. Another obvious question is, why does one want to introduce several new fermions, since a single one (for example the triplet, as in minimal DM [17] models) suffices? The answer to these questions arise from our wish to construct a model with WIMP mass at the EW scale, and hides inside the model building details, namely:

1. The off-diagonal entries of the "chargino" or "neutralino" mass matrix contain general Yukawa couplings $\left(Y_{1}\right.$ and $\left.Y_{2}\right)$ that can be enhanced as opposed to the fixed-value gauge couplings of the MSSM. Evenmore, they can be equal here i.e., $Y_{1}=Y_{2} \equiv Y \sim g$, satisfying a custodial symmetry, a realisation which is only phenomenologically allowed in the so called Split-SUSY scenarios [18, 19. Therefore, this fermionic doublet-triplet DM sector generalises the corresponding DM sector of the Minimal Supersymmetric Standard Model (MSSM).

2. In the region where the common Yukawa coupling is comparable, say, to the top Yukawa coupling there are heavy charged leptons decaying to the lightest new fermion $\chi_{1}^{0}$. This 
mass pattern, shown in Fig. 1, is different from the singlet-doublet DM model (at least from the minimal version) where the lightest neutral particle is, up to radiative corrections, degenerate with the charged particle a situation which is highly constrained from long lived charged particle searches at LHC [20].

3. In the limit of equal Yukawa couplings $(Y)$ in eq. (1.1), there is a custodial $S U(2)$ symmetry that guaranties vanishing couplings at tree level between the lightest neutral particle and the $Z$-boson $\left(Z \chi_{1}^{0} \chi_{1}^{0}\right)$ and also to the Higgs-boson $\left(h \chi_{1}^{0} \chi_{1}^{0}\right)$. This is a certain "pass" for this model, at least to leading order, from the current strong direct detection experimental contraints $[2,3,21]$. Moreover, as we shall see below, $h \chi_{1}^{0} \chi_{1}^{0}$-coupling arises radiatively at one-loop order providing us with certain model predictions. Note that "blind spots" of this kind have been studied in ref. 22] for Split-SUSY and in ref. 23] for the singlet-doublet and singlet-triplet fermionic DM models.

4. Similar to the case here, the dominant annihilation channel in the higgsino DM-phase of MSSM [24], is into gauge bosons. But in the higgsino case and due to smallness of the gauge coupling, the lightest charged and neutral fermion states are degenerate so co-annihilation effects [25] are very important. It turns out that, that for higgsino mass $\mu \sim 100 \mathrm{GeV}$ the cross section $\left\langle\sigma v>\approx \frac{g^{4}}{16 \pi \mu^{2}}\right.$ is large which results in $\Omega_{D M}$ that is too low unless $\mu$ is in the $\mathrm{TeV}$ range. In the doublet-triplet fermonic DM model we consider here, the lightest neutral state decouples from the heavy ones, and in the limit of large $m=Y v$ the difference in mass between the lightest neutral fermion and the lightest charged or the second lightest neutral one is normally of the order of $100 \mathrm{GeV}$ (see Fig. 1 for an example). The annihilation cross section now goes through the $t, u$-channels and, relative to higgsino case, is suppressed by a factor $\left(m_{\chi} / m_{\chi_{j}}\right)^{4} \sim 10-100$ where $m_{\chi_{j}}$ are the heavy fermion masses $\left(\chi_{2,3}^{0}, \chi_{1,2}^{ \pm}\right)$, allowing a WIMP mass, $m_{\chi}$, naturally of the order of $10-100 \mathrm{GeV} 3$

5. Our attempt here is to find a DM candidate particle consistent with the astrophysical and collider data but with mass around the electroweak scale. Vector-like gauge multiplets that are engaged here have also been used to construct minimal DM Models $(\mathrm{MDM})$ in Ref. [17]. It has been found that the masses $M_{D}$ or $M_{T}$ should lie in the few-TeV region. In our scenario, it is the chiral (Dirac) mass terms in eq. (1.1) that play the most important role. The latter are constrained from perturbativity to be several hundreds of $\mathrm{GeV}$ while the lower vector-like masses, $M_{D}$ and $M_{T}$, are protected by an accidental symmetry. Finally, the production and decay phenomenology of the new fermions is very distinct from the ones in MDM models and it is relatively easy to be tested with current and near future LHC data.

Within this framework of doublet-triplet fermionic DM model that we describe in Section 2 . and in particular in the region where the custodial symmetry is applied, we discuss and check constraints that include:

- An estimate of oblique corrections to electroweak observables $(S, T, U$ parameters) [Section 3 .

- DM thermal relic density calculation at tree level [Section 4].

\footnotetext{
${ }^{3}$ In this article, we are only interested in DM mass of the order of the electroweak scale.
} 
- Direct DM detection prospects through nucleus-DM particle scattering at 1-loop [Section 5

- Decay rate of the Higgs boson to two photons $(h \rightarrow \gamma \gamma)$ [Section 6

- Vacuum stability and perturbativity [Section 7

- LHC signatures, production and decays of the new fermions.

Our conclusions and various ways to extend this work are discussed in Section 9, An appendix with the explicit one-loop corrections to the $h \chi_{1}^{0} \chi_{1}^{0}$-vertex is given. Beyond the articles we have already mentioned, there is a reach literature regarding minimal DM extensions of the SM. A partial list is given in refs. 26 [6].

\section{Model Details}

As a result of what we have already mentioned in the introduction, we scan chiral fermion matter extensions of the SM gauge group according to the following, rather obvious, assumptions for the new set of fermions:

1. they must have vectorial electromagnetic interactions,

2. they must be colour singlets with integer charges,

3. their interactions must be gauge (and gravitational) anomaly free,

4. their masses are obtained after $S U(2)_{W} \times U(1)_{Y}$ gauge symmetry breaking, with only the SM Higgs doublet, and if gauge symmetry allows, directly, and

5. there is a parity symmetry, $\mathbf{Z}_{2}$, under which the SM fermions transform as +1 while the new fermions as -1 .

The most minimal model, not containing pure singlet fields ${ }^{4}$ consists of three fields arranged in colour singlets and representations of $S U(2)_{W}$, with quantum numbers denoted as $\left(\mathbf{1}^{\mathbf{c}}, \mathbf{2} \mathbf{I}_{\mathbf{W}}+\mathbf{1}\right)_{\mathbf{L}, \mathbf{R}}^{\mathbf{Y}}$, where $\mathbf{I}_{\mathbf{W}}$ is the weak $S U(2)_{W}$ isospin and $Y$ is the hypercharge related to the electric charge by $Q=I_{3 W}+\frac{Y}{2}$. These new fields are:

$$
\mathbf{T} \sim\left(1^{\mathrm{c}}, 3\right)_{\mathrm{L}}^{0}, \quad \mathrm{D}_{1} \sim\left(\mathbf{1}^{\mathrm{c}}, 2\right)_{\mathrm{R}}^{+1}, \quad \mathrm{D}_{2} \sim\left(1^{\mathrm{c}}, 2\right)_{\mathrm{R}}^{-1}
$$

One can easily check that this is a gauge and gravitational anomaly free set of chiral fermions. They sit in adjacent representations of $S U(2)_{W}$ with weak isospin difference $\Delta I_{W}=\frac{1}{2}$. This matches with the only spinless field of the SM, the Higgs field, with gauge labels $\mathbf{H} \sim\left(\mathbf{1}^{\mathbf{c}}, \mathbf{2}\right)_{+\mathbf{1}}$.

\footnotetext{
${ }^{4}$ However, see comments below.
} 
It is convenient to represent all fermions, i.e., SM quarks and leptons plus new fermions that belong to the DM sector, with two component, left handed, Weyl fields [47], namely

$$
\begin{aligned}
\text { SM quarks : } & \mathbf{Q}=\left(\begin{array}{c}
u \\
d
\end{array}\right) \sim\left(\mathbf{3}^{\mathbf{c}}, \mathbf{2}\right)_{+\mathbf{1} / \mathbf{3}}, \quad \overline{\mathbf{u}} \sim\left(\mathbf{3}^{\mathbf{c}}, \mathbf{1}\right)_{-\mathbf{4} / \mathbf{3}}, \quad \overline{\mathbf{d}} \sim\left(\mathbf{3}^{\mathbf{c}}, \mathbf{1}\right)_{+\mathbf{2} / \mathbf{3}}, \\
\text { SM leptons : } & \mathbf{L}=\left(\begin{array}{c}
\nu \\
e
\end{array}\right) \sim\left(\mathbf{1}^{\mathbf{c}}, \mathbf{2}\right)_{-\mathbf{1}}, \quad \bar{\nu} \sim\left(\mathbf{1}^{\mathbf{c}}, \mathbf{1}\right)_{\mathbf{0}}, \quad \overline{\mathbf{e}} \sim\left(\mathbf{1}^{\mathbf{c}}, \mathbf{1}\right)_{+\mathbf{2}}, \\
\text { DM fermions : } & \mathbf{T}=\left(\begin{array}{c}
T_{1} \\
T_{2} \\
T_{3}
\end{array}\right) \sim\left(\mathbf{1}^{\mathbf{c}}, \mathbf{3}\right)_{\mathbf{0}}, \\
& \overline{\mathbf{D}}_{\mathbf{1}}=\left(\begin{array}{c}
\bar{D}_{1}^{1} \\
\bar{D}_{1}^{2}
\end{array}\right) \sim\left(\mathbf{1}^{\mathbf{c}}, \mathbf{2}\right)_{-\mathbf{1}}, \quad \overline{\mathbf{D}}_{\mathbf{2}}=\left(\begin{array}{c}
\bar{D}_{2}^{1} \\
\bar{D}_{2}^{2}
\end{array}\right) \sim\left(\mathbf{1}^{\mathbf{c}}, \mathbf{2}\right)_{+\mathbf{1}} .
\end{aligned}
$$

SM fermions come in three copies of (2.4) and (2.5) sets of fields. We have added a left-handed antineutrino Weyl field in the SM field content in order to account for light neutrino masses via the seesaw mechanism. Although there may be interesting links between the neutrino and DM sector fields we shall scarcely refer to neutrinos in this article. We assume only one copy of the DM-sector fields in (2.6). Of course, we could also add more singlet fermions either in the SM or in the DM-sector but our intention is to keep the model as minimal as possible.

Physical masses are obtained from the gauge invariant form of Yukawa interactions. Under the assumption-5 above, the whole Yukawa Lagrangian of the model is

$$
\mathscr{L}_{\text {Yuk }}=\mathscr{L}_{\text {Yuk }}^{\mathrm{SM}}+\mathscr{L}_{\mathrm{Yuk}}^{\mathrm{DM}},
$$

where the SM part reads (flavour indices are suppressed):

$$
\begin{aligned}
\mathscr{L}_{\mathrm{Yuk}}^{\mathrm{SM}} & =Y_{u} \epsilon^{a b} H_{a} Q_{b} \bar{u}-Y_{d} H^{\dagger a} Q_{a} \bar{d}-Y_{e} H^{\dagger a} L_{a} \bar{e} \\
& +Y_{\nu} \epsilon^{a b} H_{a} L_{b} \bar{\nu}-\frac{1}{2} M_{N} \bar{\nu} \bar{\nu}+\text { H.c. },
\end{aligned}
$$

and the available DM-sector interactions are

$$
\begin{aligned}
\mathscr{L}_{\mathrm{Yuk}}^{\mathrm{DM}} & =Y_{1} \epsilon^{a b} T^{A} H_{a}\left(\tau^{A}\right)_{b}^{c} \bar{D}_{1 c}-Y_{2} T^{A} H^{\dagger a}\left(\tau^{A}\right)_{a}^{c} \bar{D}_{2 c} \\
& -M_{D} \epsilon^{a b} \bar{D}_{1 a} \bar{D}_{2 b}-\frac{1}{2} M_{T} T^{A} T^{A}+\text { H.c. }
\end{aligned}
$$

By choosing appropriate field redefinitions and without loss of generality we can make the parameters $Y_{1}, Y_{2}$, and $M_{T}$ real and positive, while leaving $M_{D}$ to be a general complex parameter. This is the only source of $C P$-violation 6 arising from the DM-sector in this model. If not stated otherwise, we consider real $M_{D}$ values in our numerical results. The parity symmetry assumption- 5 removes the following renormalizable operators:

$$
H^{\dagger} \bar{D}_{2} \bar{\nu}, \quad H \bar{D}_{1} \bar{\nu}, \quad L \bar{D}_{2}, \quad H T L \text { and } \quad H^{\dagger} \bar{D}_{1} \bar{e} .
$$

\footnotetext{
${ }^{5}$ The bar symbol over the Weyl fields is part of their names.

${ }^{6}$ Electron and Neutron EDMs will arise first at two-loop level. Similarly for the anomalous magnetic moments of SM leptons. See relevant discussion in ref. [48].
} 
Note that apart from the first two, the rest will not be allowed under the custodial symmetry. Finally, we assume that possible non-renormalizable operators that are allowed by the discrete symmetry are Planck scale suppressed and do not play any particular role in what follows.

\subsection{The spectrum}

Since there is no-mixing between the mass terms of the SM fermions and the DM sector ones, we solely concentrate on the non-SM Yukawa interactions of eq. (2.9). After electroweak symmetry breaking and the shift of the neutral component of the only Higgs field, $H^{0}=$ $v+h / \sqrt{2}$, we obtain the following mass terms

$$
\begin{aligned}
\mathscr{L}_{\mathrm{Y}(\text { mass })}^{\mathrm{DM}} & =-\left(\begin{array}{ll}
\tau_{1} & \bar{D}_{2}^{1}
\end{array}\right)^{T} \mathcal{M}_{C}\left(\begin{array}{c}
\tau_{3} \\
\bar{D}_{1}^{2}
\end{array}\right)-\frac{1}{2}\left(\begin{array}{lll}
\tau_{2} & \bar{D}_{1}^{1} & \bar{D}_{2}^{2}
\end{array}\right)^{T} \mathcal{M}_{N}\left(\begin{array}{c}
\tau_{2} \\
\bar{D}_{1}^{1} \\
\bar{D}_{2}^{2}
\end{array}\right)+\text { H.c. } \\
& =-\sum_{i=1}^{2} m_{\chi_{i}^{ \pm}} \chi_{i}^{-} \chi_{i}^{+}-\frac{1}{2} \sum_{i=1}^{3} m_{\chi_{i}^{0}} \chi_{i}^{0} \chi_{i}^{0}+\text { H.c. }
\end{aligned}
$$

where $\tau_{1} \equiv\left(T_{1}-i T_{2}\right) / \sqrt{2}, \tau_{3} \equiv\left(T_{1}+i T_{2}\right) / \sqrt{2}$ and $\tau_{2} \equiv T_{3}$. The charged $\left(\mathcal{M}_{C}\right)$ and the neutral $\left(\mathcal{M}_{N}\right)$ fermion mass matrices in eq. (2.11) are given by

$$
\mathcal{M}_{C}=\left(\begin{array}{cc}
M_{T} & \sqrt{2} m_{1} \\
\sqrt{2} m_{2} & -M_{D}
\end{array}\right), \quad \mathcal{M}_{N}=\left(\begin{array}{ccc}
M_{T} & m_{1} & -m_{2} \\
m_{1} & 0 & M_{D} \\
-m_{2} & M_{D} & 0
\end{array}\right),
$$

where $m_{1,2} \equiv Y_{1,2} v$. Matrices $\mathcal{M}_{C}$ and $\mathcal{M}_{N}$ are diagonalized following the singular value decomposition and the Takagi factorisation theorems [49] into $m_{\chi^{ \pm}}=(2 \times 2)$ and $m_{\chi^{0}}=(3 \times 3)$ diagonal matrices,

$$
U_{L}^{T} \mathcal{M}_{C} U_{R}=m_{\chi^{ \pm}}, \quad O^{T} \mathcal{M}_{N} O=m_{\chi^{0}}
$$

respectively, after rotating the current eigenstate fields into their mass eigenstates $\chi_{i}^{ \pm}, \chi_{i}^{0}$ with unitary matrices, $U_{L}, U_{R}$ and $O$, as

$$
\left(\begin{array}{c}
\tau_{3} \\
\bar{D}_{1}^{2}
\end{array}\right)=U_{R}\left(\begin{array}{c}
\chi_{1}^{-} \\
\chi_{2}^{-}
\end{array}\right), \quad\left(\begin{array}{c}
\tau_{1} \\
\bar{D}_{2}^{1}
\end{array}\right)=U_{L}\left(\begin{array}{c}
\chi_{1}^{+} \\
\chi_{2}^{+}
\end{array}\right), \quad\left(\begin{array}{c}
\tau_{2} \\
\bar{D}_{1}^{1} \\
\bar{D}_{2}^{2}
\end{array}\right)=O\left(\begin{array}{c}
\chi_{1}^{0} \\
\chi_{2}^{0} \\
\chi_{3}^{0}
\end{array}\right) .
$$

Therefore the spectrum of this model contains, apart from the SM masses for quarks and leptons, two additional charged Dirac fermions and three neutral Majorana particles. It is the lightest Majorana particle $\chi_{1}^{0}$ with mass $m_{\chi_{1}^{0}}$, that, perhaps, supplies the universe with cold Dark Matter.

It is crucial for what follows and also enlightening, to discuss the decoupling of the $M_{D^{-}}$ eigenvalue from the particle spectrum. First, $\mathcal{M}_{N}$, is a real symmetric matrix, under the assumption of real $M_{D}$. Then, consider the following unitary matrix $\Sigma$, having as columns orthonormal vectors,

$$
\Sigma=\frac{1}{\sqrt{2}}\left(\begin{array}{ccc}
\sqrt{2} & 0 & 0 \\
0 & 1 & 1 \\
0 & -1 & 1
\end{array}\right)
$$


which by a similarity transformation, brings the lower right $2 \times 2$ sub-block of $\mathcal{M}_{N}$ into a diagonal form,

$$
\mathcal{M}_{N}^{\prime}=\Sigma^{\dagger} \mathcal{M}_{N} \Sigma=\left(\begin{array}{ccc}
M_{T} & \left(m_{1}+m_{2}\right) / \sqrt{2} & \left(m_{1}-m_{2}\right) / \sqrt{2} \\
\left(m_{1}+m_{2}\right) / \sqrt{2} & -M_{D} & 0 \\
\left(m_{1}-m_{2}\right) / \sqrt{2} & 0 & M_{D}
\end{array}\right) .
$$

Note that since $\Sigma$ is unitary matrix, the eigenvalues of $\mathcal{M}_{N}$ and $\mathcal{M}_{N}^{\prime}$ are equal. We therefore obtain, that for $m_{1}=m_{2}$ the charged fermion mass matrix $\mathcal{M}_{C}$ becomes the upper-left subblock of the $\mathcal{M}_{N}^{\prime}$ in eq. 2.16). Therefore the eigenvalue, $M_{D}$, decouples from the neutral fermion mass matrix i.e., it is independent of any mixing and therefore any v.e.v, while the rest of eigenvalues of both matrices, $\mathcal{M}_{C}$ and $\mathcal{M}_{N}$, are one to one degenerate.

\subsection{The interactions}

We now turn to the interactions between the new fermions and the SM gauge-bosons or the SM Higgs-boson. The latter can be read from eq. (2.9) after rotating fields by exploiting the relations in 2.14. After a little bit of algebra we obtain 7

$$
\mathscr{L}_{\mathrm{Y}(\mathrm{int})}^{\mathrm{DM}}=-Y^{h \chi_{i}^{-} \chi_{j}^{+}} h \chi_{i}^{-} \chi_{j}^{+}-\frac{1}{2} Y^{h \chi_{i}^{0} \chi_{j}^{0}} h \chi_{i}^{0} \chi_{j}^{0}+\text { H.c. },
$$

where

$$
\begin{aligned}
Y^{h \chi_{i}^{-} \chi_{j}^{+}} & \equiv \frac{1}{v}\left(m_{1} U_{R 2 i} U_{L 1 j}+m_{2} U_{R 1 i} U_{L 2 j}\right), \\
Y^{h \chi_{i}^{0} \chi_{j}^{0}} & \equiv \frac{O_{1 i}}{\sqrt{2} v}\left(m_{1} O_{2 j}-m_{2} O_{3 j}\right)+(i \leftrightarrow j) .
\end{aligned}
$$

For completeness and especially for loop calculations, we append here the interactions between Goldstone bosons and the new fermions:

$$
\begin{aligned}
\mathscr{L}_{\mathrm{G} \chi \chi} & =-\frac{i O_{1 i}}{\sqrt{2} v}\left(m_{1} O_{2 j}+m_{2} O_{3 j}\right) G^{0} \chi_{i}^{0} \chi_{j}^{0}-\frac{i}{v}\left(m_{1} U_{R 2 i} U_{L 1 j}-m_{2} U_{R 1 i} U_{L 2 j}\right) G^{0} \chi_{i}^{-} \chi_{j}^{+} \\
& +\frac{m_{1}}{v}\left(\sqrt{2} U_{R 1 i} O_{2 j}-U_{R 2 i} O_{1 j}\right) G^{+} \chi_{i}^{-} \chi_{j}^{0}-\frac{m_{2}}{v}\left(\sqrt{2} U_{L 1 i} O_{3 j}+U_{L 2 i} O_{1 j}\right) G^{-} \chi_{i}^{+} \chi_{j}^{0} \\
& + \text { H.c. }
\end{aligned}
$$

Interactions among the new fermions and gauge bosons arise from the respective fermion kinetic terms. Interactions between $\chi^{ \pm}$and the photon are purely vectorial,

$$
\mathscr{L}_{\mathrm{KIN}(\mathrm{int})}^{\gamma-\chi^{ \pm}}=-(+e)\left(\chi_{i}^{+}\right)^{\dagger} \bar{\sigma}^{\mu} \chi_{i}^{+} A_{\mu}-(-e)\left(\chi_{i}^{-}\right)^{\dagger} \bar{\sigma}^{\mu} \chi_{i}^{-} A_{\mu},
$$

where $A_{\mu}$ is the photon field and $(-e)$ the electron electric charge. The $Z$-gauge boson couplings to both charged and neutral fermions can be read from 8 ,

$$
\mathscr{L}_{\mathrm{KIN}(\mathrm{int})}^{Z-\chi}=\frac{g}{c_{W}} O_{i j}^{\prime L}\left(\chi_{i}^{+}\right)^{\dagger} \bar{\sigma}^{\mu} \chi_{j}^{+} Z_{\mu}-\frac{g}{c_{W}} O_{i j}^{\prime R}\left(\chi_{j}^{-}\right)^{\dagger} \bar{\sigma}^{\mu} \chi_{i}^{-} Z_{\mu}+\frac{g}{c_{W}} O_{i j}^{\prime \prime}\left(\chi_{i}^{0}\right)^{\dagger} \bar{\sigma}^{\mu} \chi_{j}^{0} Z_{\mu},
$$

\footnotetext{
${ }^{7}$ We use Weyl notation for fermions 47 throughout.

${ }^{8}$ Our notation resembles closely the one in Appendix E of ref. 47. i.e., $U \rightarrow U_{L}^{\dagger}, V \rightarrow U_{R}^{\dagger}$ and $N \rightarrow O^{\dagger}$.
} 
where

$$
\begin{aligned}
O_{i j}^{\prime L} & =-U_{L 1 i}^{*} U_{L 1 j}-\frac{1}{2} U_{L 2 i}^{*} U_{L 2 j}+s_{W}^{2} \delta_{i j}, \\
O_{i j}^{\prime R} & =-U_{R 1 i} U_{R 1 j}^{*}-\frac{1}{2} U_{R 2 i} U_{R 2 j}^{*}+s_{W}^{2} \delta_{i j}, \\
O_{i j}^{\prime \prime L} & =\frac{1}{2}\left(O_{3 i}^{*} O_{3 j}-O_{2 i}^{*} O_{2 j}\right) .
\end{aligned}
$$

with $s_{W}, c_{W}$ the sin and cos of the weak mixing angle and $g$ the $S U(2)_{W}$ gauge coupling. Finally, interactions between $\chi$ 's and $W$-bosons are described by the terms

$$
\begin{aligned}
\mathscr{L}_{\mathrm{KIN}(\mathrm{int})}^{W^{ \pm}-\chi^{0}-\chi^{\mp}} & =g O_{i j}^{L}\left(\chi_{i}^{0}\right)^{\dagger} \bar{\sigma}^{\mu} \chi_{j}^{+} W_{\mu}^{-}-g O_{i j}^{R}\left(\chi_{j}^{-}\right)^{\dagger} \bar{\sigma}^{\mu} \chi_{i}^{0} W_{\mu}^{-} \\
& +g O_{i j}^{L *}\left(\chi_{j}^{+}\right)^{\dagger} \bar{\sigma}^{\mu} \chi_{i}^{0} W_{\mu}^{+}-g O_{i j}^{R *}\left(\chi_{i}^{0}\right)^{\dagger} \bar{\sigma}^{\mu} \chi_{j}^{-} W_{\mu}^{+},
\end{aligned}
$$

where the mixing matrices $O^{L}$ and $O^{R}$ are given by

$$
\begin{aligned}
& O_{i j}^{L}=O_{1 i}^{*} U_{L 1 j}-\frac{1}{\sqrt{2}} O_{3 i}^{*} U_{L 2 j}, \\
& O_{i j}^{R}=O_{1 i} U_{R 1 j}^{*}+\frac{1}{\sqrt{2}} O_{2 i} U_{R 2 j}^{*} .
\end{aligned}
$$

We open a parenthesis here to discuss a comparison with MSSM: mass matrices for neutral and charged fermion in eq. 2.12 remind those of neutralinos and charginos in the MSSM. It is of course trivially understood why this happens: the doublet and the triplet fields possess the same gauge quantum numbers as the higgsino and wino fields, respectively. However, there are two crucial differences: first there is no restriction to add a bino singlet and therefore the minimal $\mathcal{M}_{N}$ is a $3 \times 3$, instead of $4 \times 4$, simpler matrix and second, and more important, the off-diagonal entries in $\mathcal{M}_{N}$ and $\mathcal{M}_{C}$, are not proportional to gauge couplings but to, Yukawa couplings, $Y_{1}$ and $Y_{2}$. The latter entries $(\sim Y v)$ can be substantially bigger than the corresponding ones $(\sim g v)$ in the neutralino mass matrix of MSSM. Furthermore, since $\tan \beta=1$ is not, in general, a phenomenologically viable case in MSSM, there should always be a factor of hierarchy between the off diagonal entries. This is not necessarily the case here. In fact, the $\tan \beta=1$ "blind spot" [22], is a point in parameter space protected by a custodial symmetry.

\subsection{A custodial symmetry}

It is well known that the Higgs sector in the SM obeys, in addition to the standard electroweak gauge symmetry, a custodial $S U(2)_{R}$ global symmetry. This symmetry is broken explicitly by the hypercharge gauge coupling $g^{\prime}$, and by the difference between the top- and bottom-quark Yukawa couplings. Similarly, the fermionic DM sector, described by eq. 2.9), obeys also such a symmetry if $Y_{1}=Y_{2} \equiv Y$. More explicitly, eq. 2.9 can be written in a $S U(2)_{L} \times S U(2)_{R} \times$ $U(1)_{X}$ invariant form as

$$
\mathscr{L}_{\text {Yuk }}^{\mathrm{DM}}=-Y T^{A} \mathscr{H}^{x, a}\left(\tau^{A}\right)_{a}^{b} \overline{\mathscr{D}}_{x, b}-\frac{1}{2} M_{D} \epsilon^{x y} \epsilon^{a b} \overline{\mathscr{D}}_{x, a} \overline{\mathscr{D}}_{y, b}-\frac{1}{2} M_{T} T^{A} T^{A}+\text { H.c. },
$$


where $x, y$ denote $S U(2)_{R}$ group indices and

$$
\mathscr{H}^{x, a}=\left(\begin{array}{c}
H^{a} \\
H^{\dagger a}
\end{array}\right), \quad \overline{\mathscr{D}}_{x, a}=\left(\begin{array}{c}
\bar{D}_{1 a} \\
\bar{D}_{2 a}
\end{array}\right),
$$

with $H^{a}=\epsilon^{a b} H_{b}$. This extra global symmetry stands for the rotations between $H \leftrightarrow H^{\dagger}$ and $\bar{D}_{1} \leftrightarrow \bar{D}_{2}$. Although this symmetry is broken by the hypercharge gauge symmetry, it is natural to study interactions among extra fermions $(\overline{\mathscr{D}}, T)$ and SM-bosons under the assumption that $S U(2)_{R}$ is approximately preserved in the DM sector, that is,

$$
Y_{1}=Y_{2} \Rightarrow m_{1}=m_{2} \text {. }
$$

In addition, eq. 2.29 is invariant under a global $U(1)_{X}$ fermion number symmetry, under which only $\overline{\mathscr{D}}$ and $T$ fields are charged with $[\overline{\mathscr{D}}]=\left[D_{1}\right]=\left[D_{2}\right]=-[T]=1$. In that case $M_{D}$ and $M_{T}$ are not allowed. We therefore conclude that the limit where $Y \equiv Y_{1}=Y_{2}$ and $M_{D}=M_{T} \rightarrow 0$ is radiatively stable and this fact motivates us to study it in more detail. Note again that, both $S U(2)_{R}$ and $U(1)_{X}$ symmetries are broken explicitly by hypercharge symmetry.

\subsection{Lightest Neutral fermion interactions under the symmetry}

Let's introduce the mass difference, $\Delta m \equiv m_{1}-m_{2}$, between the chiral masses (or between Yukawa couplings, $Y_{1}$ and $Y_{2}$, if you wish). If $S U(2)_{R}$ symmetry is approximately preserved, i.e., eq. 2.30 approximately holds, $\Delta m$ must be treated as perturbation compared to $m_{1}$ or $m_{2}$ masses, which collectively denoted by $m=m_{1}$, i.e., $\Delta m \ll m$. We can then write the neutral fermion mass matrix in a suggestive perturbative form

$$
\mathcal{M}_{N}=\mathcal{M}_{N}^{(0)}+Q
$$

where

$$
\mathcal{M}_{N}^{(0)}=\left(\begin{array}{ccc}
M_{T} & m & -m \\
m & 0 & M_{D} \\
-m & M_{D} & 0
\end{array}\right), \quad Q=\left(\begin{array}{ccc}
0 & 0 & \Delta m \\
0 & 0 & 0 \\
\Delta m & 0 & 0
\end{array}\right)
$$

The zeroth order eigenvalues of $\mathcal{M}_{N}^{(0)}$ read

$$
\begin{aligned}
& m_{\chi_{1}^{0}}=M_{D}, \\
& m_{\chi_{2}^{0}}=\frac{1}{2}\left[M_{T}-M_{D}-\sqrt{8 m^{2}+\left(M_{T}+M_{D}\right)^{2}}\right], \\
& m_{\chi_{3}^{0}}=\frac{1}{2}\left[M_{T}-M_{D}+\sqrt{8 m^{2}+\left(M_{T}+M_{D}\right)^{2}}\right],
\end{aligned}
$$

while the corresponding eigenvectors are

$$
|1\rangle^{(0)}=\frac{1}{\sqrt{2}}\left(\begin{array}{l}
0 \\
1 \\
1
\end{array}\right), \quad|2\rangle^{(0)}=\frac{-1}{\sqrt{2+a^{2}}}\left(\begin{array}{c}
a \\
1 \\
-1
\end{array}\right), \quad|3\rangle^{(0)}=\frac{1}{\sqrt{2+a^{2}}}\left(\begin{array}{c}
\sqrt{2} \\
-\frac{a}{\sqrt{2}} \\
\frac{a}{\sqrt{2}}
\end{array}\right)
$$

where the parameter $a$ is given by

$$
a=\frac{m_{\chi_{1}^{0}}+m_{\chi_{2}^{0}}}{m}
$$


The parameter $a$, varies in the interval $[-\sqrt{2}, 0]$ for positive $M_{D}$. A little examination of the eigenvalues show that unless, $M_{D} \gg M_{T}>0$ where the Lightest Particle (LP) becomes the triplet, in the rest of the parameter space the LP is a "very well tempered" mixed doublet fermion, $\left|\chi_{1}^{0}\right\rangle=\frac{1}{\sqrt{2}}\left(\left|\bar{D}_{1}^{1}\right\rangle+\left|\bar{D}_{2}^{2}\right\rangle\right)$, with mass $m_{\chi_{1}^{0}}=\left.M_{D}\right|^{9}$ The DM particle $\left(\chi_{1}^{0}\right)$ has then vanishing coupling to the Higgs boson because in eq. (2.19) it is $O_{11}=0$. Note that, every neutral fermion has always vanishing diagonal couplings to $Z$-gauge boson, $\left|O_{2 i}\right|=\left|O_{3 i}\right|$, since the two doublets, $\bar{D}_{1}$ and $\bar{D}_{2}$ couple to $Z$ with opposite weak isospin. It is therefore worth examining how eigenvalues and eigenvectors are corrected after switching on to $\Delta m \neq 0$.

Obviously, in order to find how $\chi_{1}^{0}$ couples to $Z$ or $h$ non-trivially, i.e., to find the couplings $Y^{h} \chi_{1}^{0} \chi_{1}^{0}$ and $g^{Z \chi_{1}^{0} \chi_{1}^{0}}=g O_{11}^{\prime \prime L} / c_{W}$ in eqs. 2.19) and 2.25, respectively, we need to know the $O(\Delta m)$ corrections, in eigenvector the $O_{i 1}$. The corrected eigenvector, $|1\rangle=|1\rangle^{(0)}+|1\rangle^{(1)}+$ $O\left[(\Delta m)^{2}\right]$, which is nothing else but the first column of the matrix $O$ in eq. 2.13 is found to be,

$$
O_{i 1}=|1\rangle=\frac{1}{\sqrt{2}}\left(\begin{array}{c}
x \Delta m \\
1+y \Delta m \\
1-y \Delta m
\end{array}\right)+O\left[(\Delta m)^{2}\right],
$$

where

$$
\begin{aligned}
& x \equiv \frac{1}{\left(2+a^{2}\right)}\left[\frac{a^{2}}{m_{\chi_{1}^{0}}-m_{\chi_{2}^{0}}}+\frac{2}{m_{\chi_{1}^{0}}-m_{\chi_{3}^{0}}}\right], \\
& y \equiv \frac{a}{\left(2+a^{2}\right)}\left[\frac{1}{m_{\chi_{1}^{0}}-m_{\chi_{2}^{0}}}-\frac{1}{m_{\chi_{1}^{0}}-m_{\chi_{3}^{0}}}\right] .
\end{aligned}
$$

Simple substitution of eq. 2.36 into eqs. 2.19) and 2.25) gives

$$
\begin{aligned}
& Y^{h \chi_{1}^{0} \chi_{1}^{0}}=\frac{(\Delta m)^{2}}{\sqrt{2} v} x(1+2 m y)+\mathrm{O}\left[(\Delta m)^{2} / m^{2}\right], \\
& g^{Z \chi_{1}^{0} \chi_{1}^{0}} \equiv \frac{g}{c_{W}} O_{11}^{\prime \prime L}=-\frac{g}{c_{W}} y \Delta m+\mathrm{O}\left[(\Delta m)^{2} / m^{2}\right] .
\end{aligned}
$$

Obviously, for sufficiently small mass difference $\Delta m$, the Spin-Independent (SI) coupling $\left(Y^{h \chi_{1}^{0} \chi_{1}^{0}}\right)$ is suppressed by $(\Delta m)^{2} / m^{2}$ while the Spin-Dependent (SD) one $\left(g^{Z \chi_{1}^{0} \chi_{1}^{0}}\right)$ is suppressed by $\Delta m / m$ relative to their values away from the $S U(2)_{R^{-}}$symmetric limit. This maybe the reason why we have not detected DM-nucleon interactions so far. A question arises immediately about the stability of $\Delta m$ under radiative corrections. A quick RGE analysis [50,51] shows that the $\beta$-function for $\Delta m$ at 1-loop is

$$
\frac{d \Delta m}{d \ln (Q)}=\frac{\Delta m}{16 \pi^{2}}\left[\frac{29}{4} Y^{2}+3 Y_{t}^{2}-\frac{9}{20} g_{1}^{2}-\frac{33}{4} g_{2}^{2}\right]
$$

where $Y_{t}$ is the top-Yukawa coupling, $Y \equiv Y_{1} \simeq Y_{2}$, and $g_{1,2}$ the hypercharge and weak gauge couplings, respectively. Eq. 2.41 means that $\Delta m$ is only multiplicatively renormalized. Therefore, setting $\Delta m$ to zero at tree level stays zero at 1-loop and possibly at higher or$\operatorname{der}^{10}$ because this is a parameter point protected by the global symmetry. From eqs. 2.39)

\footnotetext{
${ }^{9}$ It is easy to show that since ${ }^{(0)}\langle 1|Q| 1\rangle^{(0)}=0$, there is no correction, up to $(\Delta m)^{2}$, on $m_{\chi_{1}^{0}}=M_{D} \operatorname{LP}$ mass.

${ }^{10}$ We confirm that this result remains unchanged at two-loops.
} 


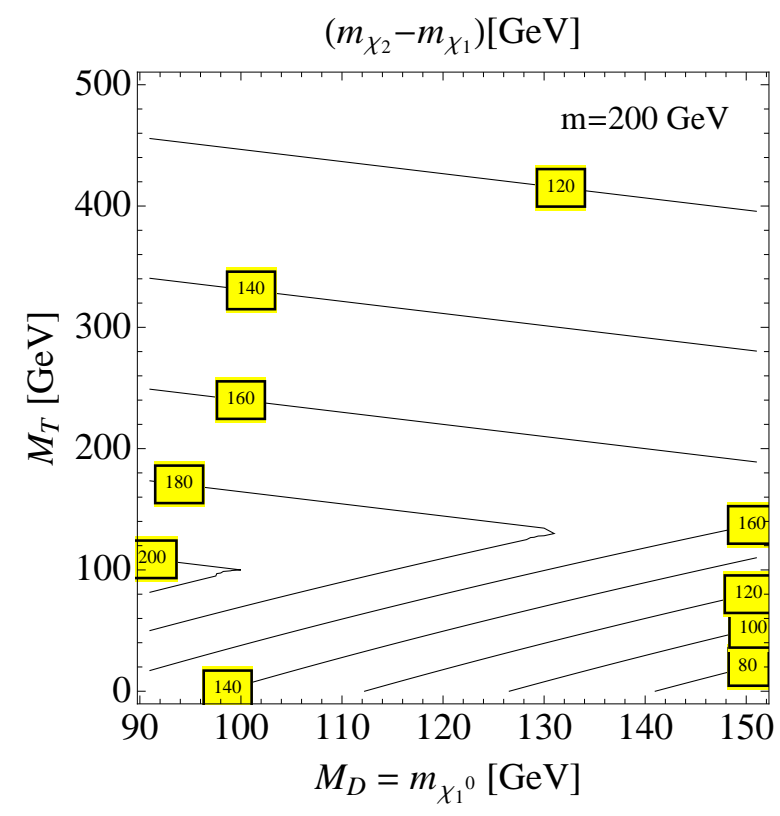

(a)

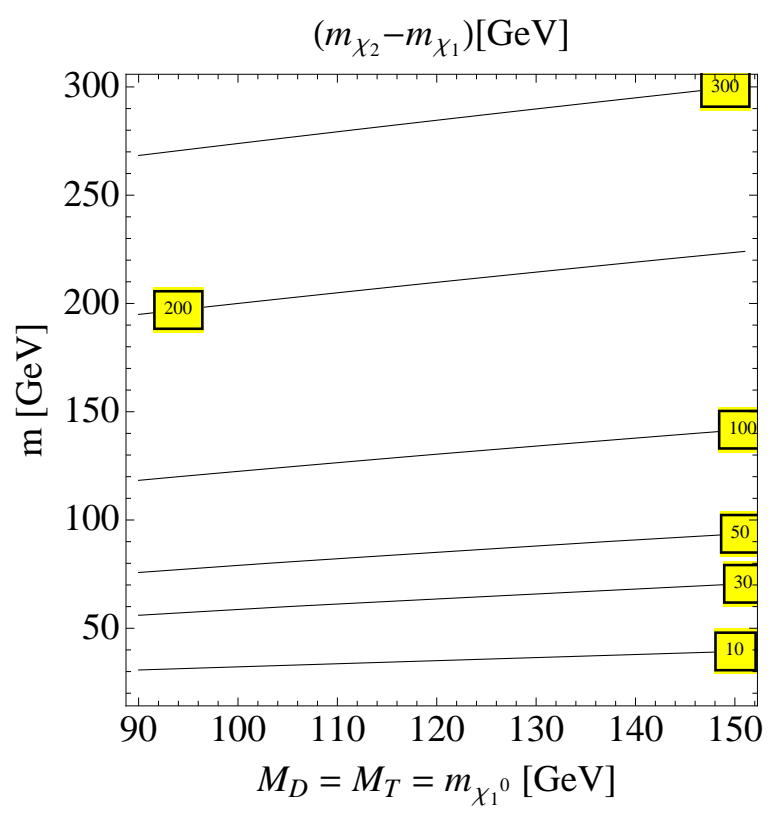

(b)

Figure 2: The mass difference, $\left|m_{\chi_{2}^{0}}\right|-\left|m_{\chi_{1}^{0}}\right|$, between the next-to-lightest and the lightest neutral particle state in the doublet-triplet fermionic DM model on (a) $M_{D}$ vs. $M_{T}$ with $m=200 \mathrm{GeV}$, and (b) on $M_{D}$ vs. $m$ with $M_{T}=M_{D}$, plane. For both plots and for the rest to come, it is always, $m_{\chi_{1}^{0}}=M_{D}$.

and (2.40) we conclude that for $\Delta m=0$, only finite (threshold) and calculable quantum corrections will affect the couplings $Y^{h \chi_{1}^{0} \chi_{1}^{0}}$ and $g^{Z \chi_{1}^{0} \chi_{1}^{0}}$ which are relevant to Direct DM searches. We confirm this consequence with a direct calculation of $\delta Y^{h \chi_{1}^{0} \chi_{1}^{0}}$ in section 5 and in Appendix A.

Note that $x$ vanishes in the limit $M_{D} \rightarrow 0$ while $(1+2 m y)$ vanishes at both $M_{D} \rightarrow 0$ and $M_{D} \rightarrow M_{T}$ limits. However, eq. 2.39 is not accurate since $(\Delta m)^{2} / m^{2}$-terms are missing in our perturbative expansion. It turns out that the $M_{D} \rightarrow M_{T}$ limit is violated by those and higher terms, but the limit $M_{D} \rightarrow 0$ is protected because of the $U(1)_{X}$-symmetry that we discussed in section 2.3. In contrast, eq. (2.40) is within $1 \%$ of its exact numerical outcome. It is also worth noticing that in the case where the Majorana masses are dominant, $M_{D}, M_{T} \gg$ $m$, then $y \rightarrow 0$ and therefore $g^{Z \chi_{1}^{0} \chi_{1}^{0}} \rightarrow 0$, up to higher order terms.

It will be useful for the discussion, especially on the relic density, to show the mass difference between the next-to-lightest $\left(\left|m_{\chi_{2}^{0}}\right|\right)$ and the lightest $\left(\left|m_{\chi_{1}^{0}}\right|\right)$ neutral fermion states. This is depicted as contour lines in Fig. $2(\mathrm{a}, \mathrm{b})$ on the $M_{D}-M_{T}$ plane (left plot) and on the $M_{D}-m$ plane with $M_{T}=M_{D}$ (right plot). Note that $M_{D}$ coincides with the LP mass i.e., $M_{D}=m_{\chi_{1}^{0}}$, everywhere in these graphs. For $m=200 \mathrm{GeV}$, the mass difference is nowhere smaller than approximately $80 \mathrm{GeV}$, and typically, it is as large as the parameter $m$ with the maximum value at $M_{D}=M_{T}$. Subsequently, in Fig. $2 \mathrm{~b}$, we plot the maximum values of the mass difference on $M_{D}-m$ plane. Alternatively, it is easy to read from Fig. 2, the parameter $a$ defined in eq. (2.35), because for the $M_{D}$ values taken throughout, it is $a=$ $-\left(\left|m_{\chi_{2}^{0}}\right|-\left|m_{\chi_{1}^{0}}\right|\right) / m$. For instance, in the plots shown, this parameter varies, approximately, 
in the region, $a \in[-1,-0.3]$.

\subsection{Analytical expressions for the new interactions under the symmetry}

As we have already discussed in section 2.1, in the symmetric $S U(2)_{R}$ limit of $(2.30)$, two of the eigenvalues from the charged fermion mass matrix are degenerate respectively with those of the neutral fermion masses given in eqs. 2.33b and 2.33c),

$$
m_{\chi_{1}^{ \pm}}=m_{\chi_{2}^{0}}, \quad m_{\chi_{2}^{ \pm}}=m_{\chi_{3}^{0}} .
$$

In addition, it is useful for further reference to present analytical expressions for all new interactions appear in the model. All these new interactions can be simply written in matrix forms containing (at most) one parameter, the real parameter $a$ of eq. 2.35). For example, rotation matrices defined in eq. (2.13) read

$$
U=U_{L}=U_{R}=\frac{1}{\sqrt{2+a^{2}}}\left(\begin{array}{cc}
a & -\sqrt{2} \\
\sqrt{2} & a
\end{array}\right), \quad O=\left(\begin{array}{ccc}
0 & -\frac{a}{\sqrt{2+a^{2}}} & \frac{\sqrt{2}}{\sqrt{2+a^{2}}} \\
\frac{1}{\sqrt{2}} & -\frac{1}{\sqrt{2+a^{2}}} & -\frac{a}{\sqrt{2} \sqrt{2+a^{2}}} \\
\frac{1}{\sqrt{2}} & \frac{1}{\sqrt{2+a^{2}}} & \frac{a}{\sqrt{2} \sqrt{2+a^{2}}}
\end{array}\right) .
$$

The couplings between $\chi_{1}^{0}, W$ and $\chi^{ \pm}$given in eq. 2.27) become explicitly:

$$
O_{1 j}^{L}=-O_{1 j}^{R *}, \quad O^{L}=\left(\begin{array}{cc}
-\frac{1}{\sqrt{2} \sqrt{2+a^{2}}} & -\frac{a}{2 \sqrt{2+a^{2}}} \\
-\frac{1+a^{2}}{2+a^{2}} & \frac{a}{\sqrt{2}\left(2+a^{2}\right)} \\
\frac{a}{\sqrt{2}\left(2+a^{2}\right)} & -\frac{4+a^{2}}{4+2 a^{2}}
\end{array}\right), \quad O^{R}=\left(\begin{array}{cc}
\frac{1}{\sqrt{2} \sqrt{2+a^{2}}} & \frac{a}{2 \sqrt{2+a^{2}}} \\
-\frac{1+a^{2}}{2+a^{2}} & \frac{a}{\sqrt{2}\left(2+a^{2}\right)} \\
\frac{a}{\sqrt{2}\left(2+a^{2}\right)} & -\frac{4+a^{2}}{4+2 a^{2}}
\end{array}\right)
$$

while those in eqs. (2.23), 2.24) and 2.25),

$$
O^{\prime L(R)}=\left(\begin{array}{cc}
\frac{-1-a^{2}+\left(2+a^{2}\right) s_{W}^{2}}{2+a^{2}} & \frac{a}{\sqrt{2}\left(2+a^{2}\right)} \\
\frac{a}{\sqrt{2}\left(2+a^{2}\right)} & -\frac{4+a^{2}-2\left(2+a^{2}\right) s_{W}^{2}}{2\left(2+a^{2}\right)}
\end{array}\right), \quad O^{\prime \prime L}=\left(\begin{array}{ccc}
0 & \frac{1}{\sqrt{2} \sqrt{2+a^{2}}} & \frac{a}{2 \sqrt{2+a^{2}}} \\
\frac{1}{\sqrt{2} \sqrt{2+a^{2}}} & 0 & 0 \\
\frac{a}{2 \sqrt{2+a^{2}}} & 0 & 0
\end{array}\right)
$$

Finally, the Higgs couplings to neutral and charged fermions in eqs. 2.19) and 2.18) are respectively:

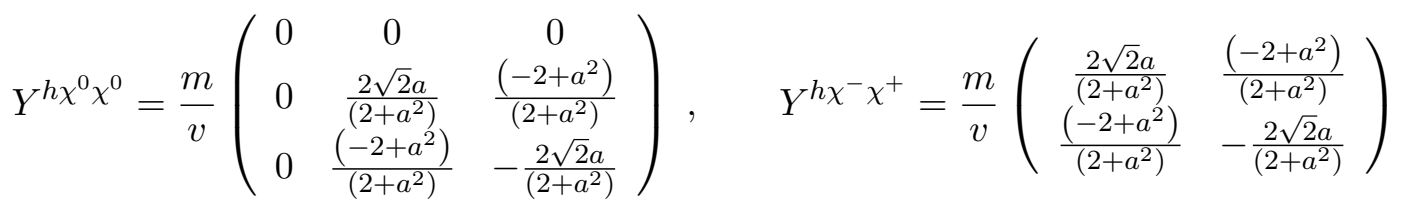

while those to Goldstone bosons given in eq. 2.20 , can now be simply written as

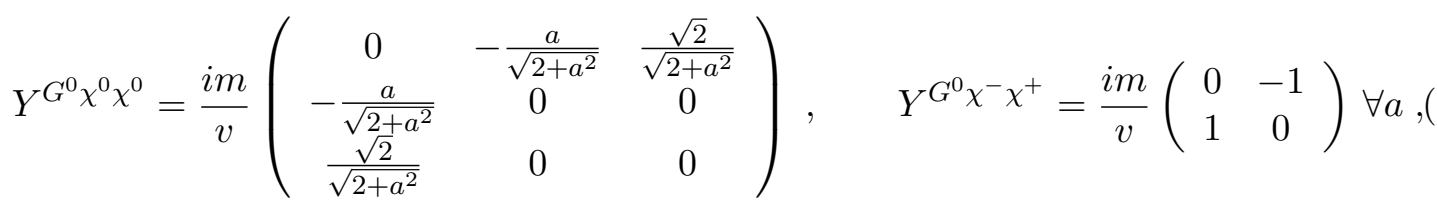

and

$$
Y^{G^{+} \chi^{-} \chi^{0}}=\frac{m}{v}\left(\begin{array}{ccc}
\frac{a}{\sqrt{2+a^{2}}} & 0 & -1 \\
-\frac{\sqrt{2}}{\sqrt{2+a^{2}}} & 1 & 0
\end{array}\right), \quad Y^{G^{-} \chi^{+} \chi^{0}}=\frac{m}{v}\left(\begin{array}{ccc}
-\frac{a}{\sqrt{2+a^{2}}} & 0 & -1 \\
\frac{\sqrt{2}}{\sqrt{2+a^{2}}} & 1 & 0
\end{array}\right) .
$$


Depending on whether the chiral mass $m$ or the vectorial masses $M_{D}$ and $M_{T}$ are dominant, and for $M_{D}>0$, there are two extreme limits for the model at hand

"Majorana dominance" $: M_{T} \approx M_{D} \gg m \Rightarrow a \approx 0, \quad m_{\chi_{1}^{0}}^{2} \approx m_{\chi_{2}^{0}}^{2} \approx M_{D}^{2}, \quad m_{\chi_{3}^{0}}^{2} \approx M_{T}^{2}$.

"Dirac dominance" : $M_{T} \approx M_{D} \ll m \Rightarrow a \approx-\sqrt{2}, \quad m_{\chi_{2}^{0}}^{2} \approx m_{\chi_{3}^{0}}^{2} \approx M_{D}^{2}+2 m^{2}$.

The "Majorana dominance" limit corresponds more or less to the "higgsino-wino" scenario of the MSSM where the first two neutral particle masses are degenerate, while the "Dirac dominance" limit is the imprint of a large Yuakawa coupling in eq. 2.9p. It is the latter case that in addition to $S U(2)_{R}$-symmetry, it is protected by the global $U(1)_{X}$ symmetry. For example, plugging in $a=-\sqrt{2}$ into eq. (2.46), we immediately see that the Higgs couplings to new fermions become diagonal resulting in a vanishing, as long as $M_{D} \rightarrow 0$, one loop corrections to the $h-\chi_{1}^{0}-\chi_{1}^{0}$ vertex, as we qualitatively confirmed in section 2.4 below eq. (2.39), and as we shall see below in section 5 .

\subsection{Composition of the lightest Neutral Fermion}

As we showed in eqs. (2.33) and (2.34), in the symmetric limit $m_{1}=m_{2}$, the neutral fermion mass matrix $\mathcal{M}_{N}$, can be diagonalized analytically into three mass eigenstates

$$
\left|\chi_{i}^{0}\right\rangle=O_{i 1}|1\rangle+O_{i 2}|2\rangle+O_{i 3}|3\rangle \text {. }
$$

Following conventional MSSM nomenclature [52], lets define the "Doublet" composition of the $\chi_{i}^{0}$ as

$$
F_{D}^{i}=\left|O_{i 2}\right|^{2}+\left|O_{i 3}\right|^{2} .
$$

Then we say that a state of $\chi_{i}^{0}$ is (D)oublet-like if $F_{D}^{i}>0.99$, it is (T)riplet like if $F_{D}^{i}<0.01$ and it is (M)ixed state if $0.01<F_{D}^{i}<0.99$.

In Fig. 3 we present the composition of the DM candidate particle $\chi_{1}^{0}$ on a $M_{D}$ vs. $M_{T}$ plane for fixed mass, $m=200 \mathrm{GeV}$. Both $Z$ and Higgs-boson couplings to pairs of $\chi_{1}^{0}$ 's vanish at tree level only in the region denoted by (D) (for Doublet) where $M_{D}$ is (most of the time) positive and equal to or less than $M_{T}$. It is mostly in this region we are focusing on in this article, because in this region the model evades, without further tweaks, direct DM detection experimental bounds. Note also that for light $M_{D}=\chi_{1}^{0} \lesssim 150 \mathrm{GeV}<<m$, the WIMP composition satisfies (D) condition for every value of $M_{T}$. For negative values of $M_{D}, \chi_{1}^{0}$ is a pure doublet only in the region $\left|M_{D}\right| \leq m$ but shrinks down to unacceptably small $M_{D}$ for large values of $M_{T}$; otherwise it is a mixed state everywhere in Fig. 3. For large $M_{D} \gg M_{T}$, the $\chi_{1}^{0}$-composition consists of mainly a triplet.

Note that when the lightest state is pure (D)oublet the heavier states are exactly an equal admixture of doublets and the triplet i.e., $F_{D}^{2,3}=0.5$.

\section{Estimate of Electroweak Corrections}

In the limit of large Yukawa couplings, $Y=Y_{1}=Y_{2} \simeq 1$, we generally expect large contributions from the new fermions, $\chi^{0}, \chi^{ \pm}$, to $(Z, W)$-gauge boson self-energy one-loop diagrams. In 


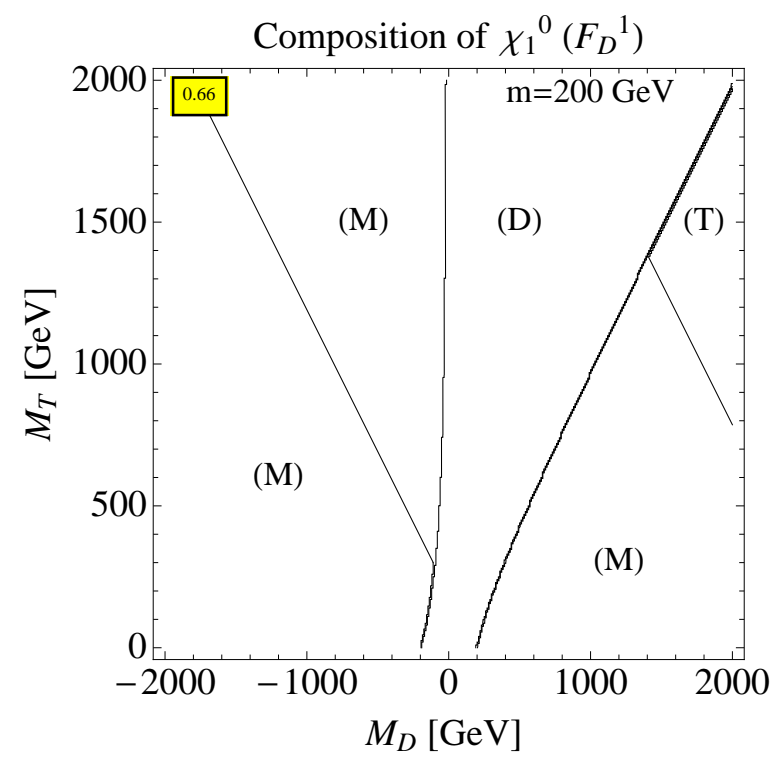

Figure 3: The composition of the WIMP in terms of (D)oublet, $(T)$ riplet and (M)ixed states following the definition given in the paragraph below eq. 2.52 , on a $M_{D}$ vs. $M_{T}$ plane and for fixed (common) Yukawa coupling, $Y=m / v \simeq 200 / 174 \simeq 1.15$.

this section we investigate constraints on the doublet-triplet fermion model parameter space, $\left\{M_{D}, M_{T}, m\right\}$, from the oblique electroweak parameters $S, T$ and $U$ [53.

Due to $Z_{2}$-parity symmetry, at one-loop level, there is no mixing between the extra fermions, $\chi^{0}, \chi^{ \pm}$, and the SM leptons. Therefore corrections to electroweak precision observables involving light fermions arise only from gauge bosons vacuum polarisation Feynman diagrams i.e., there are only oblique electroweak corrections. In order to estimate these corrections it is convenient to calculate the $S, T$ and $U$ parameters, in the limit where $m_{\chi^{0}}, m_{\chi^{ \pm}} \gtrsim m_{Z}$. This is true when the doublet mass $M_{D}$, is greater than $m_{Z}$ and $m$ is much greater than $m_{Z}$ (see Fig. 2). We shall not consider the case of a light dark matter particle, $m_{\chi_{1}^{0}} \lesssim m_{Z}$

Following closely the notation by Peskin and Takeuchi in ref. [53], we write,

$$
\begin{aligned}
\alpha S & \left.\equiv 4 e^{2} \frac{d}{d p^{2}}\left[\Pi_{33}\left(p^{2}\right)-\Pi_{3 Q}\left(p^{2}\right)\right]\right|_{p^{2}=0}, \\
\alpha T & \equiv \frac{e^{2}}{s_{W}^{2} c_{W}^{2} m_{Z}^{2}}\left[\Pi_{11}(0)-\Pi_{33}(0)\right], \\
\alpha U & \left.\equiv 4 e^{2} \frac{d}{d p^{2}}\left[\Pi_{11}\left(p^{2}\right)-\Pi_{33}\left(p^{2}\right)\right]\right|_{p^{2}=0}
\end{aligned}
$$

where $\alpha=e^{2} / 4 \pi$. In numerics we use input parameters from ref. [54], the bare value at lowest order $s_{W}^{2}=g^{\prime 2} /\left(g^{\prime 2}+g^{2}\right) \simeq 0.2312$ and the $Z$-pole mass $m_{Z}=91.1874 \mathrm{GeV}$. We calculate corrections arising only from the extra fermions, $\chi_{i=1 . .3}^{0}, \chi_{i=1 . .2}^{ \pm}$, to the $g^{\mu \nu}$ part of the gauge boson self energy amplitudes, $\Pi_{I J} \equiv \Pi_{I J}\left(p^{2}\right)$, where $I$ and $J$ may be photon $(\gamma)$, 
$W$ or $Z$,

$$
\begin{aligned}
\Pi_{\gamma \gamma} & =e^{2} \Pi_{Q Q}, \\
\Pi_{Z \gamma} & =\frac{e^{2}}{c_{W} s_{W}}\left(\Pi_{3 Q}-s^{2} \Pi_{Q Q}\right), \\
\Pi_{Z Z} & =\frac{e^{2}}{c_{W}^{2} s_{W}^{2}}\left(\Pi_{33}-2 s^{2} \Pi_{3 Q}+s^{4} \Pi_{Q Q}\right), \\
\Pi_{W W} & =\frac{e^{2}}{s_{W}^{2}} \Pi_{11},
\end{aligned}
$$

where $s_{W}=\sin \theta_{W}, c_{W}=\cos \theta_{W}$. We find,

$$
\begin{aligned}
\Pi_{Q Q} & =-\frac{p^{2}}{8 \pi^{2}} \sum_{i=1}^{2}\left[\frac{2}{3} E-4 b_{2}\left(p^{2}, m_{\chi_{i}^{ \pm}}^{2}, m_{\chi_{i}^{ \pm}}^{2}\right)\right] \\
\Pi_{3 Q} & =\frac{p^{2}}{16 \pi^{2}} \sum_{i=1}^{2}\left(Z_{i i}^{L}+Z_{i i}^{R}\right)\left[\frac{2}{3} E-4 b_{2}\left(p^{2}, m_{\chi_{i}^{ \pm}}^{2}, m_{\chi_{i}^{ \pm}}^{2}\right)\right] \\
\Pi_{33} & =\frac{1}{16 \pi^{2}} \sum_{i, j=1}^{2}\left[\left(Z_{i j}^{L} Z_{j i}^{L}+Z_{i j}^{R} Z_{j i}^{R}\right) G\left(p^{2}, m_{\chi_{i}^{ \pm}}^{2}, m_{\chi_{j}^{ \pm}}^{2}\right)-2 Z_{i j}^{L} Z_{j i}^{R} m_{\chi_{i}^{ \pm}} m_{\chi_{j}^{ \pm}} I\left(p^{2}, m_{\chi_{i}^{ \pm}}^{2}, m_{\chi_{j}^{ \pm}}^{2}\right)\right] \\
& +\frac{1}{16 \pi^{2}} \sum_{i, j=1}^{3}\left[O_{i j}^{\prime \prime} O_{j i}^{\prime \prime} G\left(p^{2}, m_{\chi_{i}^{0}}^{2}, m_{\chi_{j}^{0}}^{2}\right)+\left(O_{i j}^{\prime \prime L}\right)^{2} m_{\chi_{i}^{0}} m_{\chi_{j}^{0}} I\left(p^{2}, m_{\chi_{i}^{0}}^{2}, m_{\chi_{j}^{0}}^{2}\right)\right], \quad(3.55 \mathrm{c}) \\
\Pi_{11} & =\frac{1}{16 \pi^{2}} \sum_{i=1}^{3} \sum_{j=1}^{2}\left[\left(\left|O_{i j}^{L}\right|^{2}+\left|O_{i j}^{R}\right|^{2}\right) G\left(p^{2}, m_{\chi_{i}^{0}}^{2}, m_{\chi_{j}^{ \pm}}^{2}\right)-2 \Re e\left(O_{i j}^{L *} O_{i j}^{R}\right) m_{\chi_{i}^{0}} m_{\chi_{j}^{ \pm}} I\left(p^{2}, m_{\chi_{i}^{0}}^{2}, m_{\chi_{j}^{ \pm}}^{2}\right)\right],
\end{aligned}
$$

where $Z_{i j}^{L(R)} \equiv O_{i j}^{\prime L(R)}-s_{W}^{2} \delta_{i j}$. In addition, $E \equiv \frac{2}{\epsilon}-\gamma+\log 4 \pi-\log Q^{2}$ is the infinite part of loop diagrams. The various one-loop functions in eqs. (3.55) are given by

$$
\begin{aligned}
G\left(p^{2}, x, y\right) & =-\frac{2}{3} p^{2} E+(x+y) E+4 p^{2} b_{2}\left(p^{2}, x, y\right)-2\left[y b_{1}\left(p^{2}, x, y\right)+x b_{1}\left(p^{2}, y, x\right)\right] \\
I\left(p^{2}, x, y\right) & =2 E-2 b_{0}\left(p^{2}, x, y\right) \\
b_{0}\left(p^{2}, x, y\right) & =\int_{0}^{1} d t \log \frac{\Delta}{Q^{2}}, \quad b_{1}\left(p^{2}, x, y\right)=\int_{0}^{1} d t t \log \frac{\Delta}{Q^{2}} \\
b_{2}\left(p^{2}, x, y\right) & =\int_{0}^{1} d t t(1-t) \log \frac{\Delta}{Q^{2}}, \quad \Delta=t y+(1-t) x-t(1-t) p^{2}-i \epsilon
\end{aligned}
$$

There are numerous useful identities,

$$
\begin{aligned}
& b_{0}\left(p^{2}, x, y\right)=b_{0}\left(p^{2}, y, x\right), \quad b_{2}\left(p^{2}, x, y\right)=b_{2}\left(p^{2}, y, x\right) \\
& G\left(p^{2}, x, y\right)=G\left(p^{2}, y, x\right), \quad I\left(p^{2}, x, y\right)=I\left(p^{2}, y, x\right) \\
& b_{1}\left(p^{2}, x, y\right)=b_{0}\left(p^{2}, y, x\right)-b_{1}\left(p^{2}, y, x\right), \quad b_{1}\left(p^{2}, x, x\right)=\frac{b_{0}\left(p^{2}, x, x\right)}{2},
\end{aligned}
$$




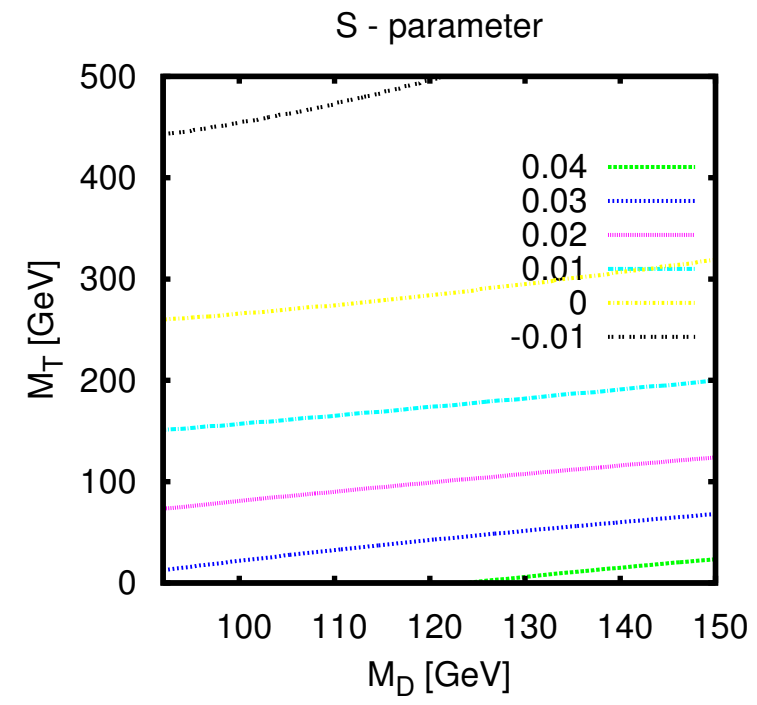

(a)

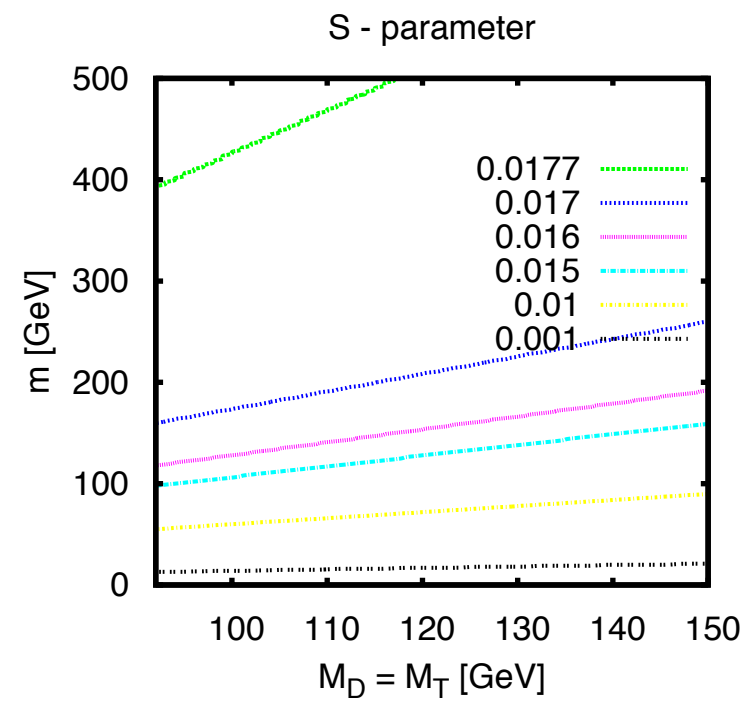

(b)

Figure 4: Contour plots of the $S$-parameter on the $M_{D}$ vs. $M_{T}$ plane (left) for $m=200 \mathrm{GeV}$ and on $M_{D}$ vs. $m$ plane (right) for $M_{T}=M_{D}$.

that will help us to simplify our expressions below. Furthermore, in the exact $S U(2)_{R}$ limit where $m_{1}=m_{2}$, there is no isospin breaking in $\bar{D}$-components and therefore $T=0$, while the $S$-parameter receives non-zero, non-decoupled, contributions due to the enlarged particle number of the $S U(2)$-sector. Specifically, in the limit where $M_{D}=M_{T} \ll m=m_{1}=m_{2}$, there is a light neutral fermion $\left(m_{\chi_{1}^{0}}\right)$ and heavy degenerate other four (two neutral and two charged) fermions, with squared mass $x$, resulting in

$$
\begin{aligned}
& \Pi_{3 Q}^{\prime}(0) \approx \frac{1}{16 \pi^{2}}\left[-2 E+2 \ln \left(\frac{x}{Q^{2}}\right)\right], \\
& \Pi_{33}^{\prime}(0)=\Pi_{11}^{\prime}(0) \approx \frac{1}{16 \pi^{2}}\left[-2 E+2 \ln \left(\frac{x}{Q^{2}}\right)+\frac{1}{18}\right], \\
& \Pi_{33}(0)=\Pi_{11}(0) \approx \frac{1}{16 \pi^{2}}\left[\frac{3 x}{2} E-\frac{3 x}{2} \ln \left(\frac{x}{Q^{2}}\right)+\frac{x}{4}\right] .
\end{aligned}
$$

Plugging in eqs. 3.63 into eqs. 3.53 we arrive at the approximate values expressions

$$
S \approx \frac{1}{18 \pi}, \quad T \approx U \approx 0 .
$$

This result is also confirmed numerically in Fig. 4 where we draw contours of the $S$-parameter on $M_{D}$ vs. $M_{T}$ plane (left plot) and on $M_{D}$ vs. $m$ plane (right plot). As it is shown, for large $m$ we obtain $S \rightarrow 1 / 18 \pi \simeq 0.0177$ while for $m \rightarrow 0$ we obtain $S \rightarrow 0$, as expected because in this case only vector-like masses will exist in $\mathscr{L}_{\mathrm{Yuk}}^{\mathrm{DM}}$ of eq. $\sqrt{2.9}$, that make no contribution to parameter $S$. Experimentally, we know [54] that when the $U$-parameter is 


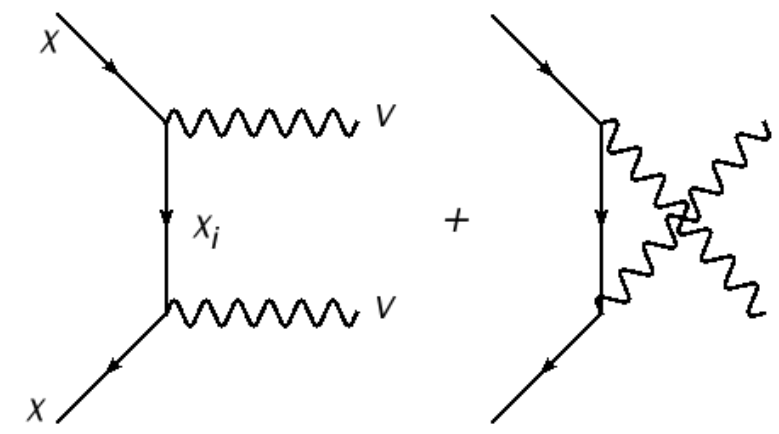

Figure 5: Lower level Feynman diagrams contributing to annihilation cross section for the process $\chi+\chi \rightarrow V+V$ for $V=W, Z$.

zero, the parameters $S$ and $T$ which fit the electroweak data are constrained to be

$$
\begin{aligned}
& S=0.04 \pm 0.09, \\
& T=0.07 \pm 0.08 .
\end{aligned}
$$

Predictions for the $S$-parameter shown in Fig. 4 a,b comfortably fall within the bound of (3.65a). In addition, even though it is not shown, the $T, U$-parameters are always negligibly small.

\section{The Thermal Relic Dark Matter Abundance}

As we have seen, $V \chi_{1}^{0} \chi_{1}^{0}$ with $V=W, Z$ and $h \chi_{1}^{0} \chi_{1}^{0}$ are forbidden at tree level if $\chi_{1}^{0}$ is a pure doublet i.e., $m_{\chi_{1}^{0}}=M_{D}$, in the exact $S U(2)_{R^{-}}$limit. Therefore, the annihilation cross section for the lightest neutral fermion results solely from the following $t$ - and $u$-channel tree level Feynman diagrams, shown in Fig. 5, with neutral or charged fermion exchange, collectively shown as $\chi_{i}$, with axial-vector interactions

$$
\begin{aligned}
& \chi_{1}^{0}+\chi_{1}^{0} \rightarrow W^{+}+W^{-}, \\
& \chi_{1}^{0}+\chi_{1}^{0} \rightarrow Z+Z .
\end{aligned}
$$

All other processes vanish at tree level. This can easily be understood by looking at the matrix forms of $O^{\prime \prime L}$ and $Y^{h \chi^{0} \chi^{0}}$ in eqs. 2.45 and 2.46). Before presenting our results for the annihilation cross section it is helpful to (order of magnitude) estimate the thermal dark matter relic density for $\chi_{1}^{0} \mathrm{~s}$. Consequently, by expanding the total cross section as $\sigma_{A n n} v=a_{V}+b_{V} v^{2}+\ldots 52,55$ and keeping only the zero-relative-velocity $a$-terms we find $\left(\right.$ for $\left.M_{D}=M_{T}\right)$ :

$$
\begin{aligned}
& a_{W}=\frac{g^{4} \beta_{W}^{3}}{32 \pi} \frac{m_{\chi}^{2}}{\left(m_{\chi}^{2}+m_{\chi_{j}}^{2}-m_{W}^{2}\right)^{2}} \underset{m \gg M_{D}}{\stackrel{m_{\chi_{j}} \gg m_{\chi}}{\longrightarrow}} \frac{g^{4} \beta_{W}^{3}}{32 \pi}\left(\frac{m_{\chi}}{m_{\chi_{j}}}\right)^{4} \frac{1}{m_{\chi}^{2}}, \\
& a_{Z}=\frac{g^{4} \beta_{Z}^{3}}{64 \pi c_{W}^{4}} \frac{m_{\chi}^{2}}{\left(m_{\chi}^{2}+m_{\chi_{j}}^{2}-m_{W}^{2}\right)^{2}} \underset{m \gg M_{D}}{\stackrel{m_{\chi_{j}} \gg m_{\chi}}{\longrightarrow}} \frac{g^{4} \beta_{Z}^{3}}{64 \pi c_{W}^{4}}\left(\frac{m_{\chi}}{m_{\chi_{j}}}\right)^{4} \frac{1}{m_{\chi}^{2}},
\end{aligned}
$$

where $g \approx 0.65$ is the electroweak coupling, $\beta_{V}=\sqrt{1-m_{V}^{2} / m_{\chi}^{2}}$ for $V=W, Z$, and in order to simplify notation, we take $m_{\chi} \equiv m_{\chi_{1}^{0}}$ to denote the DM particle mass and $m_{\chi_{j}} \equiv m_{\chi_{j}^{0}}=$ 


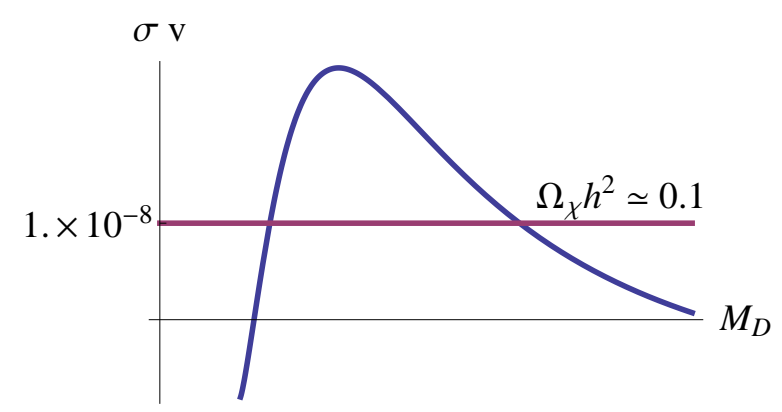

Figure 6: Sketch of the resulting annihilation cross section.

$m_{\chi_{j-1}^{ \pm}} \geq m_{\chi}$ for $j=2,3$ [see eq. 2.42 ] the heavier neutral and charged fermions of the DM-sector. In the case where $M_{D}=M_{T}$, the heavier fermions are degenerate with mass, $m_{\chi_{j}}^{2}=2 m^{2}+M_{D}^{2}$, and the mass spectrum pattern is similar to the one shown in Fig. 1. Following this pattern in eq. 4.67) we have taken the limit of $m \gg M_{D}$ or alternatively, $m_{\chi_{j}} \gg m_{\chi}$.

Obviously, eqs. 4.67a and (4.67b) viewed as functions of $M_{D}$, exhibit a maximum extremum since both $a$ 's vanish in the limits of $M_{D} \rightarrow 0$ and $M_{D} \rightarrow \infty$ and, in addition, they are positive definite. The maximum cross section is obtained approximately at $M_{D} \approx \sqrt{2} m$. The situation is clearly sketched in Fig. 6. Once again, we assume that particle- $\chi$ is a cold thermal relic, and that its mass is about few tens bigger than its freeze-out temperature. Then, universe's critical density times the Hubble constant squared (in units of $100 \mathrm{~km} / \mathrm{s} / \mathrm{Mpc}$, $\left.h^{2} \simeq 0.5\right)$ for $\chi$ 's is

$$
\Omega_{\chi} h^{2} \sim 0.1 \frac{10^{-8} \mathrm{GeV}^{-2}}{\sigma v} .
$$

Therefore, if the correct cross section, $\sigma v \approx 10^{-8} \mathrm{GeV}^{-2}$, that produces the right relic density, $\Omega_{\chi} h^{2} \sim 0.1$, happens to be below the maximum of $\sigma v$ in Fig. 6 then there are two of its points crossing the observed relic density: one for low $M_{D}$ and one for high $M_{D}$ with the single crossing point being at $M_{D} \approx \sqrt{2} \mathrm{~m}$. The mass spectrum of new fermions with high $M_{D}$ exhibits nearly degeneracy in the first two states i.e., $m_{\chi}=m_{\chi_{2}} \simeq M_{D}$. This shares similarities with the MSSM (or more precisely with the Split SUSY with $\tan \beta=1$ "winohiggsino" scenario) for higgsino Dark Matter which is well studied and we are not going to pursue further. The other case, on the other hand, with low $M_{D} \lesssim m$, exhibits a mass hierarchy between the DM candidate particle $(\chi)$ and all the rest $\left(\chi_{j}\right)$ particles. It is the suppression factor $\left(m_{\chi} / m_{\chi_{j}}\right)$ to the fourth power in eqs. (4.67a) and (4.67b) that prohibits the cross section from taking on very large values. It is therefore evident that this low $M_{D}$ scenario can provide the SM with a DM candidate particle with mass $M_{D}$ that lies "naturally" at the EW scale as this suggested by the observation $\sigma \approx 10^{-8} \mathrm{GeV}^{-2}$, and is accompanied by heavy fermions few to several times heavier (depending on the value of $m$ ) than $M_{D}$.

Before proceeding further, it is worth looking back at Fig. 2, the mass difference between the first two neutral states. For $m \gtrsim 100 \mathrm{GeV}$ the mass difference is always more than $50 \%$ than the lightest mass $m_{\chi}$. This in turn suggests that no significant contributions to $\Omega_{\chi} h^{2}$ are anticipated from co-annihilation effects [25].

In the end, we have calculated the today's relic density of the neutral, stable, and therefore, DM-candidate particle $\chi$. Our calculation is a tree level one; see however comments below. 


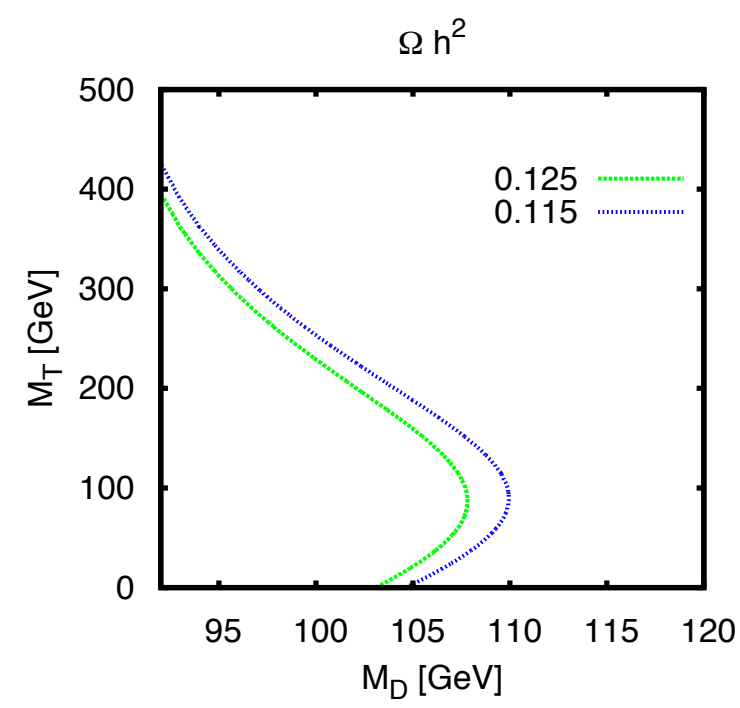

(a)

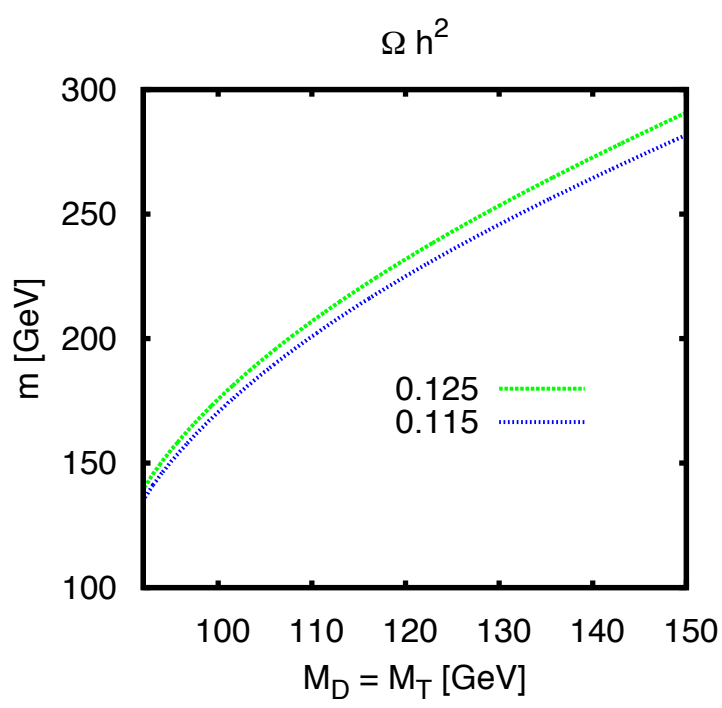

(b)

Figure 7: (Left) Contour plots on the plane $M_{D}$ vs $M_{T}$ for the observed relic density $\Omega_{\chi} h^{2}$ [see eq. 4.69)]. of the lightest neutral fermion with $m=200 \mathrm{GeV}$. (Right) The same on $M_{D}$ vs. $m$ plane for $M_{D}=M_{T}$. Recall that for both plots it is $m_{\chi}=M_{D}$.

Within the context of the (spatially flat) six-parameters standard cosmological model, Planck experiment [6] reports a density for cold, non-baryonic, dark matter, that is

$$
\Omega h^{2}=0.1199 \pm 0.0027 \text {. }
$$

The $2-\sigma$ value is satisfied only in the area between the two lines in both plots in Fig. 7. This happens for rather low $m_{\chi}=M_{D}$ in the region $92 \lesssim m_{\chi_{1}^{0}} \lesssim 110 \mathrm{GeV}$ and for $M_{T} \lesssim 420$ $\mathrm{GeV}$ on the $M_{D}-M_{T}$ plane with fixed $m=200 \mathrm{GeV}$, in Fig. 7 a 11 We also observe that the result for $\Omega_{\chi} h^{2}$ is not very sensitive to the triplet mass, $M_{T}$. Even vanishing $M_{T}$-values are in accordance with the observed $\Omega_{\chi} h^{2}$, with mass values $m_{\chi}$ laying nearby the EW scale. (If $M_{D}$ is in the region $m_{W}<M_{D}<m_{Z}$, and if we neglect three body decays, then the cross section becomes about half the one for $M_{D}>m_{Z}$. This means that $\Omega h^{2}$ is doubled and therefore larger $M_{T}$ (about twice as large) masses may be consistent with the observed $\Omega h^{2}$ values in eq. (4.69).)

We also consider the effect on $\Omega_{\chi} h^{2}$ from varying $m$ and $M_{D}$, with $M_{D}=M_{T}$, in Fig. 7 b. Obviously, the lower the $m$ is the lower the $M_{D}$ should be. For $m_{\chi} \simeq 91 \mathrm{GeV}$ the correct density is obtained for $m \simeq 140 \mathrm{GeV}$. As we move to heavier values i.e., $m \approx 300 \mathrm{GeV}, M_{D}$ (which is equal to $m_{\chi}$ ), is required to be heavier, but not much heavier, than $M_{Z}$. However, as we shall discuss in section 7 , those heavy values of $m$ are not accepted by the vacuum stability constraint without modifying the model.

\footnotetext{
${ }^{11}$ We have not considered the case $M_{D}<M_{Z}$ as this would require further three body decay analysis which is beyond the scope of this paper.
} 


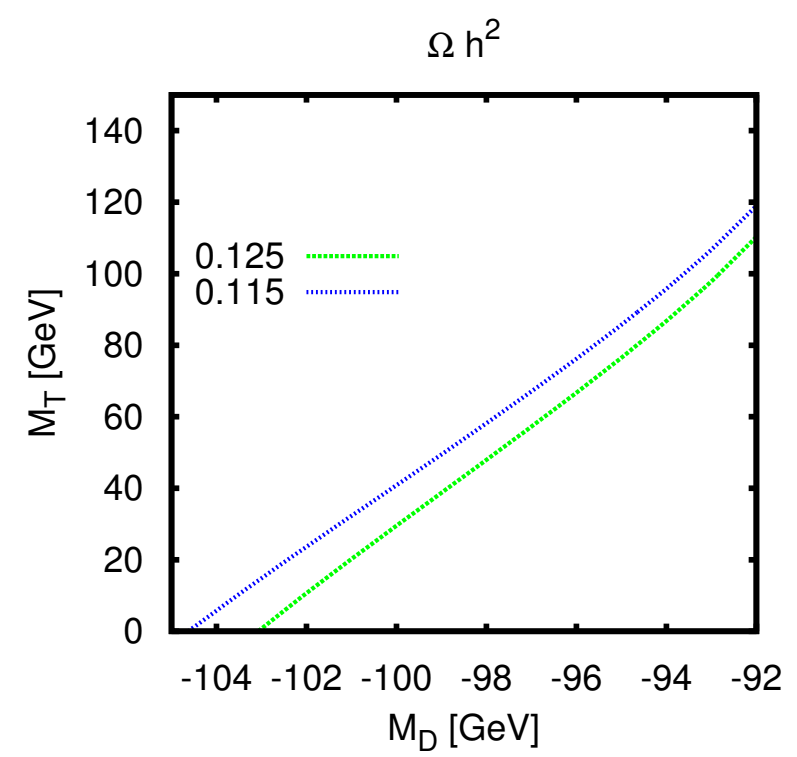

Figure 8: Same as Fig. 7 a but for negative values of $M_{D}$.

Consistent $\Omega_{\chi} h^{2}$ with observation is also achieved for negative values of $M_{D}$ in the same region as for positive $M_{D}$ as it is shown in Fig. 8. (This is the small area for negative $M_{D}$ shown in Fig. 3 where $\chi_{1}^{0}$ is Doublet). The $M_{T}$ values where this happens are limited in the mass region smaller than about $120 \mathrm{GeV}$. The EW $S$ parameter in this region is slightly moved upwards but is still consistent with eq. 3.65a. However, as we shall see below, the $M_{D}<0$ region suffers from huge suppression relative to SM in the $h \rightarrow \gamma \gamma$ decay rate.

One loop corrections to the annihilation cross section contribute only to the $b_{V}$-parameter i.e., they are $p$-wave suppressed, if $m_{\chi} \lesssim\left(m_{Z}+m_{h}\right) / 2$. Our estimate, using the crude formula of eq. 5.76 below, shows that one loop induced $b_{V}$-terms are, numerically, about 10 times smaller than the tree level ones. However, if the above limit is not hold, then ( $s$-wave) $a$ terms are coming into the final $\sigma_{A n n} v$. These terms could be of the same order as for the tree level $b$-terms and, in principle, for a precise $\Omega_{\chi} h^{2}$ prediction, they have to be included in the calculation.

We therefore conclude that, DM particle mass around the EW scale is possible and this requires large couplings of the heavy fermions to the Higgs boson i.e., large $m=Y v$ with $Y \approx 1$. and secondarily, relatively low values of triplet mass i.e., $M_{T} \simeq M_{D}$. This scenario can be hinted or completely excluded at the LHC because the couplings of the heavy new fermions (both neutral and charged) to the Higgs and gauge bosons are, in general, not suppressed in the symmetry limit [see discussion in Section 8 . 


\section{Direct DM Detection}

Following the notation of Drees and Nojiri in ref. [56], the Higgs boson mediated part of the effective Lagrangian for light quark $(u, d, s)$ - WIMP $\left(i . e\right.$. , the neutral fermion $\left.\chi_{1}^{0}\right)$ interaction is given by

$$
\mathcal{L}_{\text {scalar }}=f_{q}^{(h)} \bar{\chi}_{1}^{0} \chi_{1}^{0} \bar{q} q
$$

Note that in this model there are no tensor contributions (at 1-loop level) since $\chi_{1}^{0}$ does not interact directly with coloured particles (as opposed to supersymmetric neutralino for example). The next step is to form the nucleonic matrix elements for the $\bar{q} q$ operator in eq. (5.70) and we write

$$
\left\langle n\left|m_{q} \bar{q} q\right| n\right\rangle=m_{n} f_{T q}^{(n)}
$$

where $m_{n}=0.94 \mathrm{GeV}$, is the nucleon mass. The form factors $f_{T q}^{(n)}$ are obtained within chiral perturbation theory and the experimental measurements of pion-nucleon interaction term, and they are subject to significant uncertainties. $f_{T q}^{(n)}$ for $q=u, d[57 \mid$ are generically small by, say, a factor of $O(10)$ compared to $f_{T s}=0.14$ obtained from ref. 58 value which we adopt into our numerical findings here. However, bear in mind that $f_{T s}$ is subject to large theoretical errors [52,57]. For instance, the average value quoted from lattice calculations [59 is $0.043 \pm 0.011$, which is smaller by a factor of three from the one obtained from chiral perturbation theory. This will result in, at least, a factor of $\mathcal{O}(10)$ reduction in the WIMPnucleon cross section results, presented in Fig. 9, below.

The Higgs boson couples to quarks and then to gluons through the one-loop triangle diagram. Subsequently, the gluons $(G)$ couple to the heavy quark current through the heavy quarks $(Q=c, b, t)$ in loop. The analogous $(q \rightarrow Q)$ matrix element in eq. 5.70 for $m_{Q} \bar{Q} Q$ can be replaced by the trace anomaly operator $-\left(\alpha_{s} / 12 \pi\right) G \cdot G$ to obtain

$$
\left\langle n\left|m_{Q} \bar{Q} Q\right| n\right\rangle=\frac{2}{27} m_{n}\left[1-\sum_{q=u, d, s} f_{T q}^{n}\right] \equiv \frac{2}{27} m_{n} f_{T G} .
$$

We are ready now to write down the effective couplings of $\chi_{1}^{0}$ to nucleons $(n=p, n)$ :

$$
\frac{f_{n}}{m_{n}}=\sum_{q} \frac{f_{q}^{(h)}}{m_{q}} f_{T q}^{(n)}+\frac{2}{27} \sum_{Q} \frac{f_{Q}^{(h)}}{m_{Q}} f_{T G} .
$$

Note that the bigger the $f_{T s}$ is, the bigger the $f_{n}$ becomes. Also note that $f_{q}^{(h)} \propto m_{q}$. Furthermore, for $f_{T s} \simeq 0.14$ the second term in eq. (5.73), which is formally a two loop contribution to $f_{n}$, is about a factor of two smaller than the first one. Under the above assumption for the $f_{T s}$ dominance we obtain $f_{p}=f_{n}$. In this case, the Spin Independent (SI) elastic scattering cross section at zero momentum transfer, of the WIMP $\chi_{1}^{0}$ scattering off a given target nucleus with mass $m_{N}$ in terms of the coupling $f_{p}$ is

$$
\sigma_{0(\text { scalar })}=\frac{4}{\pi} \frac{m_{\chi_{1}^{0}}^{2} m_{N}^{4}}{\left(m_{\chi_{1}^{0}}+m_{N}\right)^{2}}\left(\frac{f_{p}}{m_{n}}\right)^{2} .
$$

The perturbative dynamics of the model is contained in the factor $f_{p}$ and therefore, from eq. 5.73), in $f_{q}^{(h)}$ and $f_{Q}^{(h)}$. In this particular model the form factor $f_{q}^{(h)}$ reads,

$$
\frac{f_{q}^{(h)}}{m_{q}}=\frac{g\left[\Re e\left(Y^{h \chi_{1}^{0} \chi_{1}^{0}}\right)-\delta Y^{h \chi_{1}^{0} \chi_{1}^{0}}\right]}{4 m_{W} m_{h}^{2}} .
$$




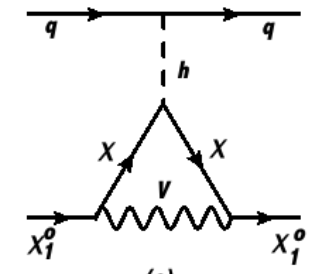

(a)

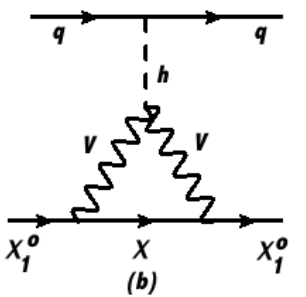

(b)

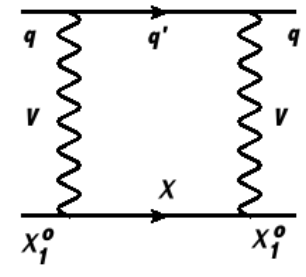

(c)

Figure 9: Feynman diagrams (in unitary gauge) related to spin independent (SI) elastic cross section $\chi_{1}^{0}+q \rightarrow \chi_{1}^{0}+q$ where $q=u, d, s$ - light quarks. Particle $V$ represents $W$ or $Z$ and $\chi$ represents $\chi_{i=1 . .2}^{ \pm}$or $\chi_{i=1 . .3}^{0}$, respectively. One loop self energy corrections are absent in the particular scenario we have chosen.

The Higgs coupling to lightest neutral fermions is given in eq. (2.19). In particular, under the custodial symmetry consideration we adopt here, it is obvious from eq. (2.46), that $Y^{h \chi_{1}^{0} \chi_{1}^{0}}=0$, at tree level. Generic one-loop corrections will be proportional to, $g^{2} Y / 4 \pi \approx 0.03$, which can easily fall in the experimental exclusion region from current direct experimental DM searches for large $Y \sim 1$ coupling (see for instance eq.(3) in ref. [22]). We therefore need to calculate the one loop corrections, $\delta Y^{h} \chi_{1}^{0} \chi_{1}^{0} \equiv \delta Y$ to the $h \chi_{1}^{0} \chi_{1}^{0}$-vertex.

There is a fairly quick way to get an order of magnitude reliable calculation of $\delta Y$ through the Low Energy Higgs Theorem (LEHT) [60 63]. Application of LEHT in the region of our interest i.e., $m_{\chi_{1}^{0}} \approx m_{W} \approx m_{h} \ll m_{\chi_{i}^{ \pm}}$or $M_{D} \approx M_{T} \approx m_{W} \ll m$, and considering only Goldstone boson contributions to $\chi_{1}^{0}$ one-loop self energy diagrams, results in

$$
\delta Y=\frac{\partial}{\partial v} \delta M_{D}(v) \approx \frac{Y^{3}}{4 \pi^{2}} \frac{M_{D} m}{M_{D}^{2}+2 m^{2}}, \quad M_{D} \approx M_{T} \approx m_{W} \approx m_{h} \ll m .
$$

Let's inspect eq. (5.76). First, the middle term explains trivially why the Higgs coupling is zero at tree level: the lightest eigenvalue of the neutral mass matrix is $M_{D}$ which is independent on any vacuum expectation value (v.e.v.). Then because at one loop, the $\chi_{1}^{0}$ self-energies involve only the heavy fermion masses (both charged and neutral) which depend on the v.e.v through $m=Y v$ or through $m_{W}, m_{Z}$ in the propagators of $\chi_{i}^{ \pm}, \chi_{i=2,3}^{0}$ and $W, Z$ respectively, the one loop correction $\delta Y$ does not in general vanish. Second, the third term of the equality eq. (5.76) shows that the effect increases by the third power of the Yukawa coupling $Y$ [recall eq. (2.28)] and vanishes when $M_{D} \rightarrow 0$ [the $U(1)_{X}$ symmetry limit]. As for the numerical approximation, eq. (5.76) is always less than $20 \%$ of the exact calculation (see below) even though we have completely neglected the non-Goldstone diagrams that are proportional to gauge couplings. It is however a crude approximation which is only relevant when the new heavy fermions are far heavier than the $Z, W, h$-bosons as well as from the lightest neutral fermion.

In Appendix A we calculate the exact one-loop amplitude for the vertex $h-\chi_{1}^{0}-\chi_{1}^{0}$ with physical external $\chi_{1}^{0}$ particles at a zero Higgs-boson momentum transfer. A similar calculation has been carried out in ref. [64] for the MSSM and in ref. [65] for minimal DM models. However, due to peculiarities of this model that have been stressed out in the introduction with respect to the aforementioned models, a general calculation is needed. The one-loop corrected vertex amplitude arises from (a) and (b) diagram ${ }^{12}$ depicted in Fig. 9 involving

\footnotetext{
${ }^{12}$ Note that, Eq. 2.46 implies that there are no self energy contributions to - $i \delta Y$ - at one-loop.
} 
vector bosons $(W$ or $Z)$ and new charged $\left(\chi_{i=1,2}^{ \pm}\right)$or neutral $\left(\chi_{i=1 . .3}^{0}\right)$ fermions, as

$$
i \delta Y=\sum_{j=(a),(b)}\left(i \delta Y_{j}^{\chi^{ \pm}}+i \delta Y_{j}^{\chi^{0}}\right) .
$$

Detailed forms, not resorting to $C P$-conservation, for $\delta Y$ 's are given in Appendix A. We have proven both analytically and numerically that when the external particles $\chi_{1}^{0}$ are on-shell, infinities cancel in the sum of the two vertex diagrams in Fig. 9a,b without the need of any renormalization prescription, and the resulting amplitude - $i \delta Y$ - is finite and renormalization scale invariant.

We have also carried out the one-loop calculation of the box diagrams in Fig. 9c. The effective operators for box diagrams consist of scalar, $f_{q}^{(\text {box })}$ [like the $f_{q}$ in eq. (5.70)] and twist operators, $g_{q}^{(1)}$ and $g_{q}^{(2)}$ written explicitly for example in ref. $[56$. In the parameter space of our interest where $M_{D} \ll m$, the $f_{q}^{(\text {box })}$ contributions to $f_{q}^{(\bar{h})}$ in eq. 5.73 , are in general two orders of magnitude smaller than the vertex ones arising from Fig. $9(\mathrm{a}, \mathrm{b})$, and they are only important in the case where the latter cancel out among each other. Moreover, it has recently been shown in refs. 66 68] that, the full two-loop gluonic contributions are relevant for a correct order of magnitude estimate of the cross section in the heavy WIMP mass limit, especially when adopting the "lattice" value for $f_{T_{s}}$. We are not aware, however, of any study dealing with those corrections and WIMP mass around the electroweak scale which is the case of our interest. Such a calculation is quite involved and is beyond the scope of the present article.

In Fig. 10 we present our numerical results for the SI nucleon-WIMP cross section. The current LUX 3 (XENON100 2]) experimental bounds for a $100 \mathrm{GeV}$ WIMP mass is $\sigma_{0}^{(S I)} \lesssim$ $1(2) \times 10^{-45} \mathrm{~cm}^{2}$ at $90 \%$ C.L.

From the left panel of Fig. 10 we observe that in the region where $M_{T} \ll M_{D} \ll m$ the cross section is by one to two orders of magnitude smaller than the current experimental bound. More specifically, in the region where we obtain the right relic density [see Fig. 77] the prediction for the $\sigma_{0}^{(S I)}$ is about to be observed only for large values of $M_{T}\left(M_{T} \approx 500\right.$ $\mathrm{GeV})$, while it is by an order of magnitude smaller for low values of $M_{T}\left(M_{T} \lesssim 100 \mathrm{GeV}\right)$. There is a region, around $M_{T} \approx 25 \mathrm{GeV}$, where box corrections, that arise from the diagram in Fig. 9k, on scalar and twist-2 operators become important because the vertex corrections mutually cancel out. However, in this region the cross-section becomes two to four orders of magnitude smaller than the current experimental sensitivity. We also remark that $\sigma_{0}^{(S I)}$ reaches a maximum value, indicated by the closed contour line in the upper left corner of Fig. 10a, and then starts decreasing for larger $M_{T}$ and $M_{D}$ values, a situation that looks like following the Appelquist-Carazzone decoupling theorem [69]. However, even at very large masses, $M_{D}$ and $M_{T}$, not shown in Fig. 10, there is a constant piece of $\delta Y$, and hence of $\sigma_{0}^{(S I)}$, that does not decouple. This can be traced respectively in the second and the first terms of integrals $I_{4}^{V}$, and $I_{5}^{V}$ of eq. A.7), in the limit $M_{D}=M_{T} \rightarrow \infty$. This non-decoupling can also been seen in the heavy particle, effective field theory analysis of ref. [66] and also in refs. [17, 65]. We have also checked numerically that $\sigma_{0}^{(S I)}$ vanishes at $M_{D} \rightarrow 0$ as expected from eq. (5.76) and from the $U(1)_{X}$-symmetry ${ }^{13}$

\footnotetext{
${ }^{13}$ Because only $\bar{D}_{1,2}$ are charged under $U(1)_{X}$ (not the Higgs boson), and $\chi_{1}^{0}$ is a linear combination of only $\bar{D}$ 's.
} 


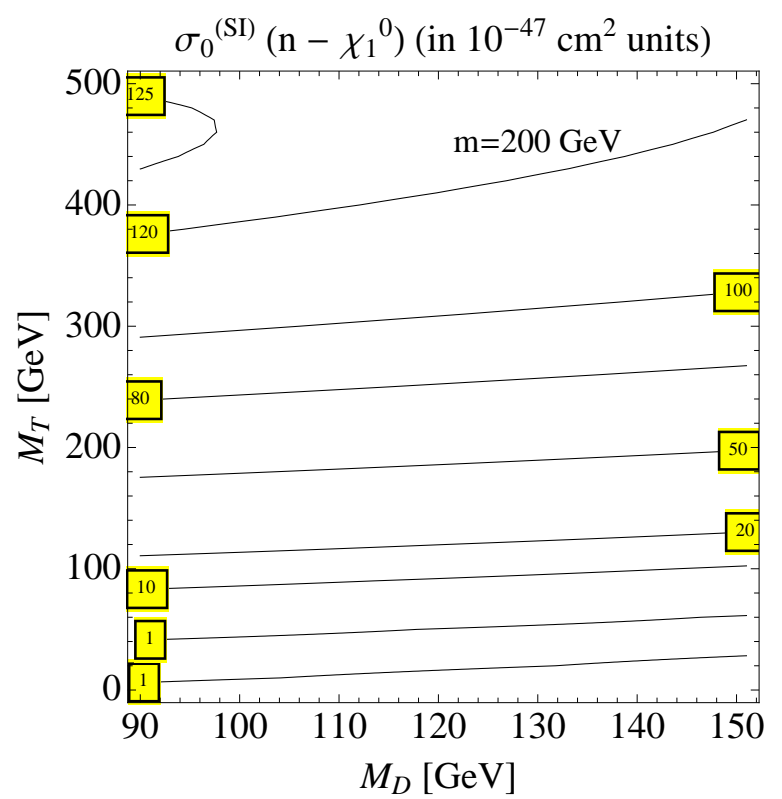

(a)

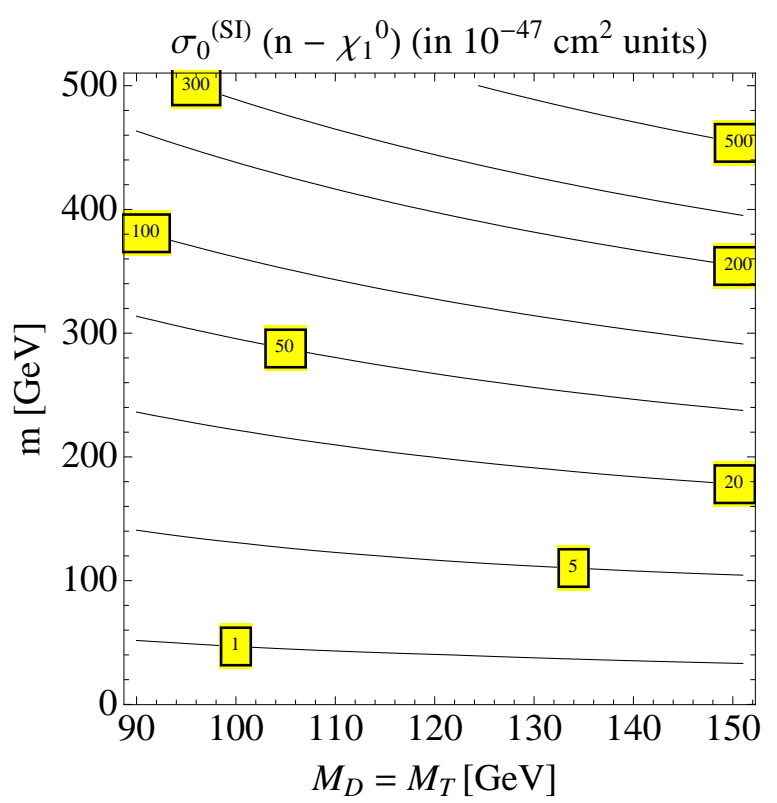

(b)

Figure 10: Results (in boxed labels) for the Spin-Independent (SI) scattering cross section for the nucleon - WIMP (n- $\left.\chi_{1}^{0}\right)$ in units of $10^{-47} \mathrm{~cm}^{2}$ on a $M_{D}$ vs. $M_{T}$ plane for fixed parameter $m=Y v=200 \mathrm{GeV}$ (left) and on $M_{D}$ vs. $m$ plane for fixed $M_{T}=M_{D} G e V$ (right).

In Fig. 10p, we also plot predictions for the doublet-triplet fermionic model on SI cross section $\sigma_{0}^{(S T)}$ on $M_{D}$ vs. $m$ plane for $M_{T}=M_{D}$. As we recall from eq. (5.76), the cross section increases with $m$ (or $Y$ ) as $m^{2} \propto Y^{2}$. It becomes within current experimental sensitivity reach for $m \gtrsim 400 \mathrm{GeV}$ while for low $m \approx 100 \mathrm{GeV}, \sigma_{0}^{(S I)}$ is about 100 times smaller. Besides, for heavy $M_{D}$ and $m$ (upper right corner), $\sigma_{0}^{(S I)}$ becomes excluded by current searches although vacuum stability bounds hit first. If we compare with the corresponding plot for the relic density in Fig. $7 \mathrm{~b}$, we see that the observed $\Omega_{\chi} h^{2}$ is allowed by current experimental searches on $\sigma_{0}^{(S I)}$ but it will certainly be under scrutiny in the forthcoming experiments [4].

Finally, for negative values of $M_{D}$ consistent with the observed density depicted in Fig. 8 , it turns out that $\sigma_{0}^{(S I)}$ is by a factor of about $\sim 10$ bigger than the corresponding parameter space for $M_{D}>0$ given in Fig. 10a. In fact, the region of 1-loop cancellations happened for $M_{T} \approx 20 \mathrm{GeV}$, do not take place for $M_{D}<0$. However, within errors discussed at the beginning of this section, this is still consistent with current experimental bounds.

\section{$6 \quad$ Higgs boson decays to two photons}

In the doublet-triplet fermionic model there are two pairs of electromagnetically charged fermions and antifermions, namely, $\chi_{1}^{ \pm}, \chi_{2}^{ \pm}$. They have electromagnetic interactions with charge $Q= \pm 1$ and interactions with the Higgs boson, $Y^{h \chi^{-} \chi^{+}}$, given in general by eqs. 2.17) and (2.18), or in particular, in the symmetry limit, by eq. 2.46). These latter interactions are of similar size as of the top-quark-antiquark pairs with the Higgs boson i.e., $Y \sim 1$. Hence, we expect a substantial modification of the decay rate, $\Gamma(h \rightarrow \gamma \gamma)$ relative to the SM 


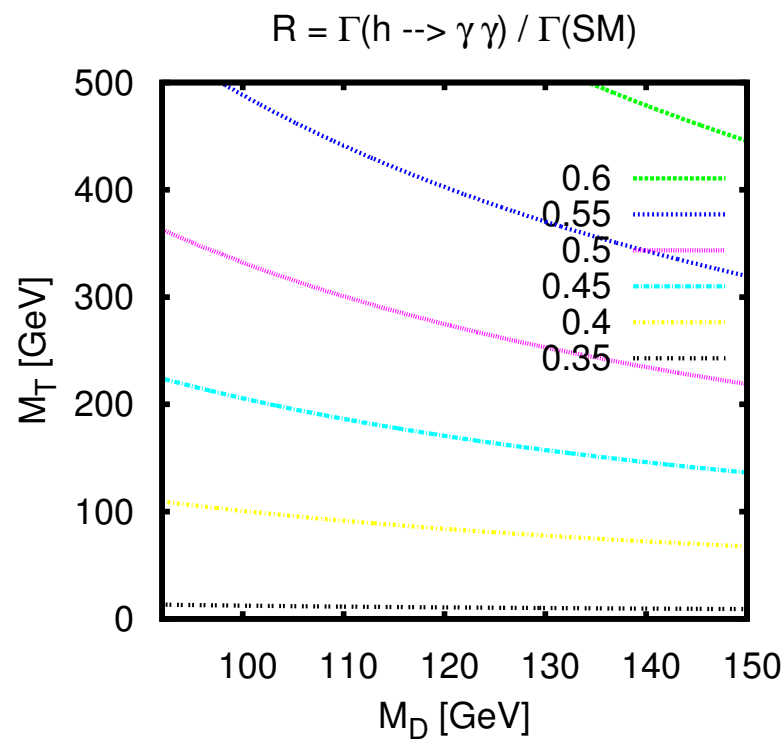

(a)

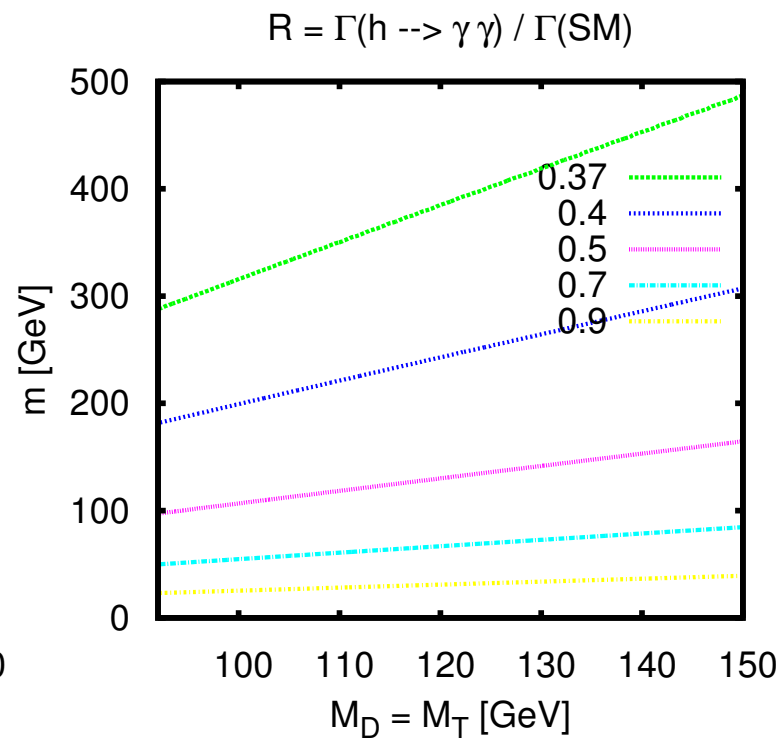

(b)

Figure 11: Contour lines for the ratio, $R=\Gamma(h \rightarrow \gamma \gamma) / \Gamma(h \rightarrow \gamma \gamma)_{\mathrm{SM}}$, for the decay rate of Higgs boson decays into two photons over the $S M$ prediction on (a) $M_{D}$ vs. $M_{T}$ plane with $m=200 \mathrm{GeV}$ and (b) on $M_{D}$ vs. $m$ plane with $M_{T}=M_{D}$.

onf ${ }^{14} \Gamma(h \rightarrow \gamma \gamma)_{S M}$, through the famous triangle graph [60], involving $W$-gauge bosons, the top-quark $(t)$ and the new fermions $\chi_{i}^{ \pm}$. Under the assumption of real $M_{D}, Y^{h \chi_{i}^{-} \chi_{i}^{+}}$is also real, and we obtain:

$$
R \equiv \frac{\Gamma(h \rightarrow \gamma \gamma)}{\Gamma(h \rightarrow \gamma \gamma)_{(\mathrm{SM})}}=\left|1+\frac{1}{A_{\mathrm{SM}}} \sum_{i=\chi_{1}^{ \pm}, \chi_{2}^{ \pm}} \sqrt{2} \frac{Y^{h \chi_{i}^{-} \chi_{i}^{+}} v}{m_{\chi_{i}^{+}}} A_{1 / 2}\left(\tau_{i}\right)\right|^{2},
$$

where $A_{\mathrm{SM}} \simeq-6.5$ for $m_{h}=125 \mathrm{GeV}$, is the SM result dominated by the $W$-loop [70], with $\tau_{i}=m_{h}^{2} / 4 m_{i}^{2}$ and $A_{1 / 2}$ is the well known function given for example in ref. 71 15, The $\chi_{i}^{ \pm}$-fermion contribution $\left(Q=1, N_{c}=1\right)$, is also positive because the ratio, $Y^{h \chi_{i}^{-}} \chi_{i}^{+} / m_{\chi_{i}^{+}}$, is always positive when $m_{\chi_{1}^{0}}=M_{D}$, as can be seen by inspecting eqs. 2.46, 2.50) and 2.33 . After using the simplified (by symmetry) eq. 2.46 with $a \approx-\sqrt{2}$, we approximately obtain

$$
\sum_{i} \frac{\sqrt{2} m}{m_{\chi_{i}^{+}}} A_{1 / 2}\left(\tau_{\chi_{i}^{+}}\right) \approx+\frac{8}{3}
$$

which means that $\Gamma(h \rightarrow \gamma \gamma)$ is smaller than the SM expectation. But how much smaller? In Fig. 11 we plot contours of the ratio $R \equiv \Gamma(h \rightarrow \gamma \gamma) / \Gamma(h \rightarrow \gamma \gamma)_{(\mathrm{SM})}$ on $\left(M_{D}\right.$ vs. $\left.M_{T}\right)$-plane

\footnotetext{
${ }^{14}$ The Higgs boson production cross section is the same with the SM because the new fermions are uncoloured.

${ }^{15}$ The Higgs-fermion vertex is parametrized here as $\mathcal{L} \supset-Y h \bar{f} f+$ h.c. and therefore for the top-quark Yukawa we obtain $Y_{i} \rightarrow Y_{t} / \sqrt{2}$ from eq. 2.8) while for the new charged fermions $Y_{i} \rightarrow Y^{h \chi_{i}^{-}} \chi_{i}^{+}$from eqs. 2.17. and 2.18 .
} 
for $m=200 \mathrm{GeV}$ (Fig $11 \mathrm{a})$ and $M_{D}$ vs. $m$-plane for $\left(M_{T}=M_{D}\right)$ (Fig 11b). Our numerical results plotted in Fig. 11 are exact at one-loop. We observe that the new charged fermions render the ratio less than unity

$$
R \lesssim 1
$$

everywhere in the parameter space considered. Let's look at this in a more detail. The contribution of fermions $\chi_{i}^{ \pm}$in eq. 6.78), depends on the quantity ${ }^{16}$

$$
\sim \frac{2 m^{2}}{2 m^{2}+M_{D} M_{T}}
$$

which is always positive for $M_{D}, M_{T}>0$ i.e., it adds to the top-quark contribution and subtracts from the large and negative $W$-boson one resulting in a suppressed $R$-ratio. If instead we choose $M_{D}<0$, then for $\left|M_{D} M_{T}\right|>\sqrt{2}|m|$, one can obtain $R \gtrsim 1$, a situation which is explored in ref. [51]. As can be seen from Fig. 3 however, in this case the DM candidate particle $\left(\chi_{1}^{0}\right)$ is not a pure doublet. It is instead a mixed state. (In fact the states $|1\rangle$ and $|2\rangle$ are interchanged in eq. (2.34)). As a consequence, there is a non-zero (and generically large) $h \chi_{1}^{0} \chi_{1}^{0}$-coupling already present at tree level, and, bear in mind fine tuning, it is excluded by direct DM search bounds.

By comparing areas with the observed relic density in Figs.7(a,b) we see that, the results for $0.35 \lesssim R \lesssim 0.5$ shown in Figs. 11(a,b) are within $1 \sigma$-error compatible with current central values of CMS measurements $73(0.78 \pm 0.27)$ but are highly "disfavoured" by those from ATLAS 74 ones, $1.65 \pm 0.24(\text { stat })_{-0.18}^{+0.25}($ syst $)$. The forthcoming second LHC run will be decidable in favour or against this outcomes here.

Fig. 11(a) or eq. 6.81), shows also that when $M_{T}$ becomes heavy the ratio $R$ approaches the current CMS central value. This happens because one of the two charged fermion eigenvalues becomes very heavy, $m_{\chi_{2}^{+}} \approx M_{T}$, and therefore it is decoupled from the ratio. As we discussed in section 4 , large $M_{T} \sim 1 \mathrm{TeV}$ values, may be consistent with the observed $\Omega_{\chi} h^{2}$ for $m_{W}<M_{D}<m_{Z}$. We have found that even in this case, $R$ is always smaller than 0.65.

If we assume that $M_{D}<0$ and $\chi_{1}^{0}$ pure doublet as shown in Fig. 3 , then it is always $R<1$. In fact, using the input values from Fig. 8 for the correct relic density, the suppression of $R$ is even higher, $0.25 \lesssim R \lesssim 0.35$. Alternatively, if we assume that $M_{D}$ is a general complex parameter, then the coupling, $Y^{h \chi_{1}^{-}} \chi_{1}^{+}$, is complex too. In this case one has to add the CP-odd Higgs contribution into eq. 6.78) which is always positive definite. For large phases relatively large $M_{T}$ the ratio $R$ may be greater than one, however, again the direct detection bounds are violated by a factor of more than 10-1000.

Of course, if we increase $M_{D}$, the parameter space may be compatible with the observed relic density seen in the right side of "heavy" $M_{D}$-branch in Fig. 6. However, following our motivation for "only EW scale DM" we do not discuss this region further which is anyhow very well known from MSSM studies.

We therefore conclude that in the doublet triplet fermionic model thermal DM relic abundance for low DM particle mass $m_{\chi_{1}^{0}} \approx M_{Z}$, consistent with observation [6] and with direct DM searches [2.3] leads to a substantial suppression (45-75\%) for the rate $\Gamma(h \rightarrow \gamma \gamma)$ relative to the SM expectation.

\footnotetext{
${ }^{16}$ This quantity is obtained also by using the low energy Higgs theorem as in ref. 72 for the singlet-doublet DM-case.
} 


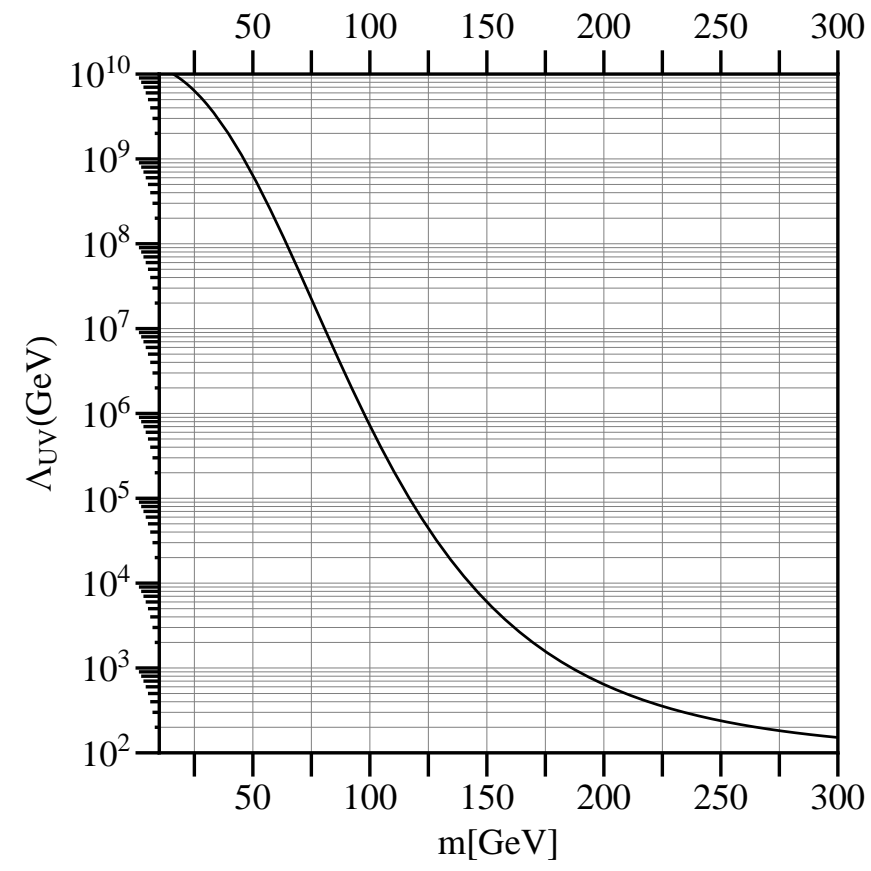

Figure 12: The vacuum stability plot: $\Lambda_{U V}$ against $m=Y v$.

We have also calculated the ratio $R$ for the Higgs boson decay into $Z \gamma$. The results are similar to the case of $R(h \rightarrow \gamma \gamma)$. In particular, in the parameter space explored in Fig. 11(a), we observe exactly the same shape of lines with a ratio slightly shifted upwards in the region, $0.4 \lesssim R(h \rightarrow Z \gamma) \lesssim 0.7$. This suppression is due to the same reason discussed in the paragraph below eq. 6.81 .

\section{Vacuum Stability}

The stability of the Standard Model vacuum is an important issue, so we need to find an energy scale $\left(\Lambda_{U V}\right)$ where new physics is needed, in order to make the vacuum stable or a metastable (unstable with lifetime larger than the age of the universe). To make an estimate about the $\Lambda_{U V}$ of the theory, one needs to calculate the tunnelling rate between the false and the true vacuum and impose that the SM vacuum has survived until today ${ }^{17}$. Following ref. [76], we can see that the bound for the Higgs self coupling, $\lambda$, becomes ${ }^{18}$,

$$
\lambda\left(\Lambda_{U V}\right)=\frac{4 \pi^{2}}{3 \ln \left(\frac{H}{\Lambda_{U V}}\right)},
$$

where $\Lambda_{U V}$ is the cut off scale and $H$ is the Hubble constant $H=1.5 \times 10^{-42} \mathrm{GeV}$. In order to impose the contstraint 7.82 , we also need to find the running parameter $\lambda$ by solving the renormalization group equations. The one-loop beta functions for the model at hand are

\footnotetext{
${ }^{17}$ The probability of the tunnelling has been calculated at tree level in ref. 75 .

${ }^{18}$ This bound can also be found in ref. 51 .
} 
given in ref. $50,51,77[19$, and we solve this set of differential equations using as initial input parameters:

$$
\begin{aligned}
\alpha_{3}\left(M_{Z}\right) & =0.1184, \quad \alpha_{2}\left(M_{Z}\right)=0.0337, \quad \alpha_{1}\left(M_{Z}\right)=0.0168 \\
\lambda\left(M_{Z}\right) & =0.1303, \quad y_{t}\left(M_{Z}\right)=0.9948, \quad M_{Z}=91.1876 \mathrm{GeV} .
\end{aligned}
$$

The result for the cut off scale as a function of $m=Y v$ is given in Fig. 12. As we can see, $\Lambda_{U V} \approx 600 \mathrm{GeV}$ for $m \approx 200 \mathrm{GeV}$ which is quite small while $\Lambda \approx 20 \mathrm{TeV}$ for $m \approx 130 \mathrm{GeV}$. The result for $\Lambda_{U V}$ in Fig. 12 is only approximate. Threshold effects, from the physical masses of the doublet, triplet and even the top-quark, together with comparable two-loop corrections to $\beta$-functions, which can be found for example in refs. [50, 77], are missing in Fig. 12. These effects may change the outcome for $\Lambda_{U V}$ by a factor of two or so but they will not change the conclusion, that extra new physics is required already nearby the TeV-scale. The form of new physics will probably be in terms of new scalar fields since extra new fermions will make $\Lambda_{U V}$ even smaller. These scalars may be well within reach at the second run of the LHC [51] but it is our assumption here that they do not intervene with the DM sector.

As far as the (1-loop) perturbativity of the Yukawa couplings $Y \sim 1.2$ (for $m=200$ ) and $Y_{t}$, is concerned, these exceed the value $4 \pi$ at around the respective scales, $10^{9}$ and $10^{10}$ $\mathrm{GeV}$. Given the modifications of the model that must be performed at $\Lambda_{U V} \sim \mathrm{TeV}$ scale, the perturbativity bound is of secondary importance here.

\section{Heavy fermion production and decays}

The unknown new fermions that have been introduced into this model to accompany the DM mechanism can be searched for at the LHC in a similar fashion as for charginos and neutralinos of the MSSM. Multilepton final states associated with missing energy may arise in three different ways from the decays of new fermion pairs: $\chi_{i}^{+} \chi_{j}^{-}, \chi_{i}^{ \pm} \chi_{j}^{0}$, and $\chi_{i}^{0} \chi_{j}^{0}$.

\subsection{Production}

A recent study at LHC 78,79 has presented upper limits in the signal production cross sections for charginos and neutralinos, in the process

$$
p+p \rightarrow W^{*} \rightarrow \chi_{1}^{+}+\chi_{2}^{0},
$$

which is mediated by the $W$-gauge boson. One can use Fig. $9 \mathrm{~b}$ from ref. [78] to set limits to the cross section and therefore to constrain the parameter space. This figure fits perfectly into our study since it assumes a) $100 \%$ branching ratio for the $\chi_{1}^{+}$and $\chi_{2}^{0}$ decays as it is the case here [see section 8.2 below] b) degenerate masses for $\chi_{1}^{+}$and $\chi_{2}^{0}$ as it is exactly the case here as shown in eq. (2.42). The production cross section has been calculated in ref. [80] also including next to leading order QCD corrections. The parton-level, tree level, result is

$$
\frac{d \hat{\sigma}}{d \hat{t}}\left(u+d^{\dagger} \rightarrow W^{*} \rightarrow \chi_{i}^{+}+\chi_{j}^{0}\right)=\frac{1}{16 \pi \hat{s}^{2}}\left(\frac{1}{3 \cdot 4} \sum_{\text {spins }}|\mathcal{M}|^{2}\right),
$$

\footnotetext{
${ }^{19}$ We need to make the substitutions $\tilde{g}_{2 d} \rightarrow-Y_{1}$ and $\tilde{g}_{2 u} \rightarrow-Y_{2}$ because of different conventions with ref. 50$]$.
} 


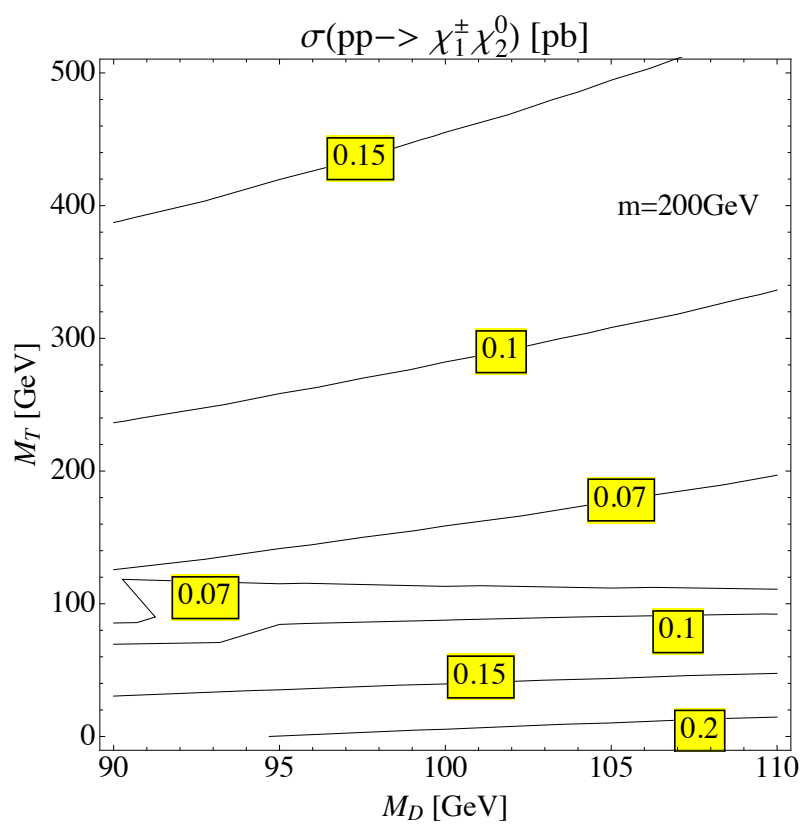

Figure 13: Contours of the production cross section for the new fermions, $\sigma\left(p p \rightarrow \chi_{1}^{ \pm} \chi_{2}^{0}\right)$ [in $p b]$, on $M_{D}$ vs. $M_{T}$ plane, at $L H C$ with $\sqrt{s}=8 \mathrm{TeV}$.

where the factors $1 / 3$ and $1 / 4$ arise from colour and spin average of initial states, $\hat{s}, \hat{t}, \hat{u}$ are the Mandelstam variables at the parton level, and

$$
\sum_{\text {spins }}|\mathcal{M}|^{2}=\left|c_{1}\right|^{2}\left(\hat{u}-m_{\chi_{i}^{+}}^{2}\right)\left(\hat{u}-m_{\chi_{j}^{0}}^{2}\right)+\left|c_{2}\right|^{2}\left(\hat{t}-m_{\chi_{i}^{+}}^{2}\right)\left(\hat{t}-m_{\chi_{j}^{0}}^{2}\right)+2 \Re e\left[c_{1} c_{2}^{*}\right] m_{\chi_{i}^{+}} m_{\chi_{j}^{0}} \hat{s}(8.8 .87)
$$

with the coefficients $c_{i}$ being

$$
c_{1}=-\frac{\sqrt{2} g^{2}}{\hat{s}-m_{W}^{2}} O_{j i}^{L *}, \quad c_{2}=-\frac{\sqrt{2} g^{2}}{\hat{s}-m_{W}^{2}} O_{j i}^{R *} .
$$

We let the indices $i=1,2$ and $j=1,2,3$ free as there is a situation of a complete mass degeneracy between the heavy neutral and charged fermions when $M_{D}=M_{T}$. Our result in eqs. 8.86) and (8.87) are in agreement with refs. 47, 80.

By convoluting eq. 8.86 with the proton's pdfs and integrating over phase space we obtain in Fig. 13, the production cross section for $\sigma\left(p p \rightarrow \chi_{1}^{ \pm} \chi_{2}^{0}\right)$ [in $\left.p b\right]$. In the region with correct DM relic density, we obtain typical values varying in the interval $(0.07-0.2) p b$ for $\sqrt{s}=8 \mathrm{TeV}$. This is about 1400-4000 events at LHC before any experimental cuts assuming $20 \mathrm{fb}^{-1}$ of accumulated luminosity. This is within current sensitivity search and analysis has been performed by ATLAS [78] and CMS 79 for simplified supersymmetric models. Looking for example in Fig. 9b in ATLAS [78, for the same parameter space as in our Fig. 13, the observed upper limit on the signal cross section varies in the interval (0.14-1.2) $p b$. In the region where $M_{D}=M_{T}$, all heavy fermions are mass degenerate. In this case the total cross section is the sum of all possible production modes $\chi_{1,2}^{ \pm} \chi_{2,3}^{0}$, and the total cross section is about $0.15 p b$ which is on the spot of current LHC sensitivity $(0.14 \mathrm{pb})$ [78]. 


\subsection{Decays}

Just by looking at a typical spectrum of the model in Fig. 1, we see that the heavy fermions can decay on-shell to two final states with a gauge boson and the lightest neutral stable particle. Therefore, the lightest charged and the next to lightest neutral fermions decay like

$$
\begin{gathered}
\chi_{1}^{ \pm} \rightarrow \chi_{1}^{0}+W^{ \pm}, \\
\chi_{2}^{0} \rightarrow \chi_{1}^{0}+Z .
\end{gathered}
$$

In our case where $\chi_{1}^{0}$ is a "well tempered doublet" there are no-off diagonal couplings to the Higgs boson, like for example $h \chi_{1}^{0} \chi_{2}^{0}$. Therefore, particles $\chi_{1}^{ \pm}$and $\chi_{2}^{0}$ decay purely to final states following $8.88 \mathrm{a}$ ) and $8.88 \mathrm{~b}$ ) with $100 \%$ branching fractions. The signature at hadron colliders is the well know from SUSY searches, trileptons plus missing energy.

Analytically we find the decay widths 47,81 :

$$
\begin{aligned}
\Gamma\left(\chi_{i}^{+} \rightarrow \chi_{j}^{0}+W^{+}\right) & =\frac{g^{2} m_{\chi_{i}^{+}}}{32 \pi} \lambda^{1 / 2}\left(1, r_{W}, r_{j}\right)\left\{\left(\left|O_{j i}^{L}\right|^{2}+\left|O_{j i}^{R}\right|^{2}\right)\left[1+r_{j}-2 r_{W}+\left(1-r_{j}\right)^{2} / r_{W}\right]\right. \\
& \left.-12 \sqrt{r_{j}} \Re e\left(O_{j i}^{L *} O_{j i}^{R}\right)\right\}, \\
\Gamma\left(\chi_{i}^{0} \rightarrow \chi_{j}^{0}+Z\right) & =\frac{g^{2} m_{\chi_{i}^{0}}}{16 \pi c_{W}^{2}} \lambda^{1 / 2}\left(1, r_{Z}, r_{j}\right)\left\{\left|O_{i j}^{\prime \prime}\right|^{2}\left[1+r_{j}^{0}-2 r_{Z}+\left(1-r_{j}^{0}\right)^{2} / r_{Z}\right]\right. \\
& +6 \sqrt{r_{j}^{0}} \Re e\left[\left(O_{i j}^{\prime \prime}{ }^{2}{ }^{2}\right]\right\},
\end{aligned}
$$

where

$$
\begin{gathered}
r_{W} \equiv m_{W}^{2} / m_{\chi_{i}^{+}}^{2}, \quad r_{Z} \equiv m_{Z}^{2} / m_{\chi_{i}^{0}}^{2}, \quad r_{j} \equiv m_{\chi_{j}^{0}}^{2} / m_{\chi_{i}^{+}}^{2}, \quad r_{j}^{0} \equiv m_{\chi_{j}^{0}}^{2} / m_{\chi_{i}^{0}}^{2} \\
\lambda(x, y, z) \equiv x^{2}+y^{2}+z^{2}-2 x y-2 x z-2 y z .
\end{gathered}
$$

Numerical results for the decay widths for the processes $88.88 \mathrm{a})$ and $8.88 \mathrm{~b})$ in the area of interest are depicted in Fig. 14(a) and (b), respectively. Both decay widths behave similarly. In the area $M_{D} \approx M_{T} \approx 100 \mathrm{GeV}$ we observe maximum values $\Gamma \approx 3 \mathrm{GeV}$. As $M_{T}$ is increases or decreases, the widths get smaller than $1 \mathrm{GeV}$. This is easily understood if we look back at the mass difference $\left|m_{\chi_{2}^{0}}\right|-\left|m_{\chi_{1}^{0}}\right|$ in Fig. 2(a) and recall that for the parameter considered in Fig. 14, it is $m_{\chi_{1}^{0}}=M_{D}$ and $m_{\chi_{2}^{0}}=m_{\chi_{1}^{ \pm}}$.

For heavier charged fermions, new decay channels include

$$
\begin{aligned}
& \chi_{2}^{+} \rightarrow \chi_{1}^{+}+Z, \\
& \chi_{2}^{+} \rightarrow \chi_{1}^{+}+h,
\end{aligned}
$$

that are mostly kinematically allowed in the low $M_{D} \approx 100 \mathrm{GeV}$ but high $M_{T} \gtrsim 220 \mathrm{GeV}$ regime. For the heavier neutral particles, if kinematically allowed they would decay to $W, Z$ gauge bosons and/or the Higgs boson,

$$
\begin{aligned}
& \chi_{3}^{0} \rightarrow \chi_{1}^{ \pm}+W^{\mp}, \\
& \chi_{3}^{0} \rightarrow \chi_{2}^{0}+Z, \\
& \chi_{3}^{0} \rightarrow \chi_{2}^{0}+h .
\end{aligned}
$$




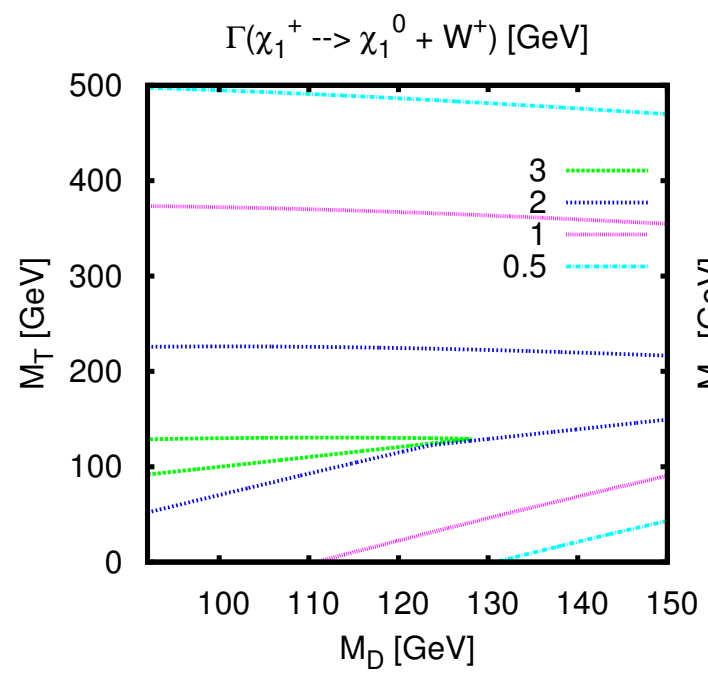

(a)

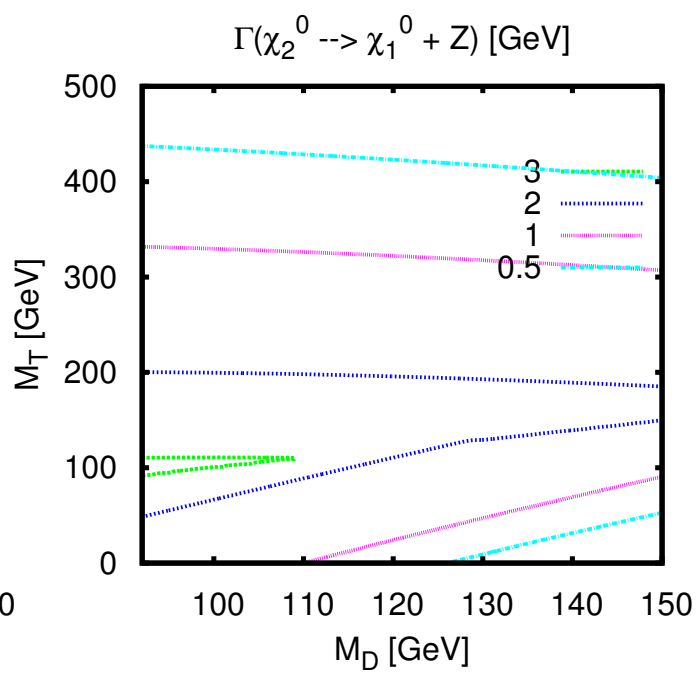

(b)

Figure 14: Contour plots for the decay rates [in GeV] for the processes $\chi_{1}^{+} \rightarrow \chi_{1}^{0}+W^{+}$(left) and $\chi_{2}^{0} \rightarrow \chi_{1}^{0}+Z$ (right). We assume $m=200 \mathrm{GeV}$.

\section{Conclusions and Future Directions}

Our motivation for writing this paper is to import a simple DM sector in the SM with particles in the vicinity of the electroweak scale responsible for the observed DM relic abundance, preferably not relying on co-annihilations or resonant effects, and capable of escaping current detection from nucleon-recoil experiments. Meanwhile, we study consequences of this model in EW observables and Higgs boson decays $(h \rightarrow \gamma \gamma, Z \gamma)$ and other possible signatures at LHC.

This SM extension consists of two fermionic $S U(2)_{W}$-doublets with opposite hypercharges and a fermionic $S U(2)_{W}$-triplet with zero hypercharge. The new interaction Lagrangian is given in eq. (2.9), and contains both Yukawa trilinear terms together with explicit mass terms for the doublets and triplet fields. Under the assumption of a certain global $S U(2)_{R^{-}}$ symmetry, discussed in section 2.3 , that rotates $H$ to $H^{\dagger}$ and $\bar{D}_{1}$ to $\bar{D}_{2}$, the two Yukawa couplings become equal with certain consequences that capture our interest throughout this work. After electroweak symmetry breaking this sector widens the SM with two charged Dirac fermions and three neutral Majorana fermions, the lightest $\left(\chi_{1}^{0}\right)$ of which plays the role of the DM particle. Under the symmetry assumption and for Yukawa couplings comparable to top-quark, the lightest neutral particle $\left(\chi_{1}^{0}\right)$ may have mass equal to the vector-like mass of the doublets, $M_{D}$, and its field composition contains only an equal amount of the two doublets [see Fig. 3]. As a result, the couplings of the Higgs and the $Z$ bosons to the lightest neutral fermion pair vanish at tree level.

Within this framework we observe in Fig. 7 , that $\Omega_{\chi} h^{2}$, is in accordance with observation [eq. 4.69]] provided that the parameters of the model, $M_{D}, M_{T}$ and $m$, lie naturally at the 
EW scale i.e., without the need for resonant or co-annihilation effects. Moreover, the $\chi_{1}^{0}-$ nucleon SI cross section appears at one-loop, turns out to be around 1-100 times smaller than the current experimental sensitivity from LUX and XENON1T as it is shown in Fig. 10. In addition, we find that the oblique electroweak parameters $S, T$ and $U$ are all compatible with EW data fits as it is shown in Fig. 4, a result which is partly a consequence of the global symmetry exploited.

We also look for direct implications at the LHC. We find that the existence of the extra charged fermions reduces substantially the ratios of the Higgs decay to di-photon (see Fig. 11) and to $Z \gamma$ w.r.t the SM. This is a certain prediction of this scenario that cannot be avoided by changing the parameter space. For very large Yukawa coupling, this reduction maybe of up to $65 \%$ relative to the SM expectation as we obtain from Fig. 11. Furthermore, the production and decays of those new charged/neutral fermion states, is within current and forthcoming LHC reach. Decay rates for some of these states are shown in Fig. 14.

We should notice here that the minimality of the Higgs sector together with the $Z_{2}$-parity symmetry preserves the appearance of new flavour changing or CP-violating effects beyond those of the SM, for up to two-loop order (for a nice discussion of effects on EDMs from the charged fermions, see ref. [48]).

On top of collider/astrophysical constraints, we made an estimate of the consequences of the new states to vacuum stability of the model. The 1-loop result for the UV cutt off scale, above which the model needs some completion, is given in Fig. 12 . We see that for the parameter space of interest, new physics, probably in the form of new, supersymmetric, scalars is needed already nearby the $\mathrm{TeV}$ or multi-TeV scale to cancel fermionic contributions in the quartic Higgs coupling. For example, this solution may take the form of an MSSM extension with $\bar{D}_{1,2}$ and $T$ superfield (extensions with a triplet superfield have been explored in ref. [82]).

In summary, in this work we basically studied the synergy between three observables: $\Omega_{\chi} h^{2}, \sigma_{0}^{S I}$, and $R(h \rightarrow \gamma \gamma)$, in a simple fermionic DM model. If charged fermion states are discovered at the second run of LHC and are compatible with $\Omega_{\chi} h^{2}$ with $m_{\chi} \sim m_{Z}$, then $R(h \rightarrow \gamma \gamma)$ has to be suppressed i.e., $R$ will turn towards the CMS central value. If instead $R(h \rightarrow \gamma \gamma) \gtrsim 1$ is enhanced, then the DM particle is heavy, $m_{\chi} \sim 1 \mathrm{TeV}$, or otherwise excluded by direct DM detection bounds. If $R \sim 1$, then one has to go to large $M_{T}$ values where, however, $\Omega_{\chi} h^{2}$ is only barely compatible with $m_{\chi} \simeq m_{Z}$. In this latter case, the mass of the DM particle may be below the EW gauge boson masses. However, in this case an entire new analysis is required.

Apart from studying the regime with mass $m_{\chi}$ lower than $M_{Z}$, this work can be extended in several ways as for example, to investigate the role of $C P$-violating phases of $M_{D}$ on baryogenesis. Indirect DM searches could be also an interesting avenue together with extensions of the Higgs sector. We postpone all these interesting phenomena for future study.

\section{Acknowledgements}

We are grateful to Susanne Westhoff for helping us fiinding a mistake in our formulae in the Appendix A. Our results for nucleon-WIMP cross section, depicted in Fig. 10, are now in good agreement with ref. [83]. A. D. would like to thank, M. Drees for useful comments, A. Barucha for discussions on the ongoing LHC chargino searches, C. Wagner for drawing 
our attention to ref. [13], and F. Goertz for discussions on custodians. We are grateful to J. Rosiek for letting us using his code for squaring matrix elements and to compare with our analytical results. Our numerical routines for matrix diagonalization and for loop functions follow closely those in refs. 84, 85, respectively.

This research Project is co-financed by the European Union - European Social Fund (ESF) and National Sources, in the framework of the program "THALIS" of the "Operational Program Education and Lifelong Learning" of the National Strategic Reference Framework (NSRF) 2007-2013. D.K. acknowledges full financial support from the research program "THALIS".

\section{Appendix A}

The 1-loop corrected vertex amplitude arises from (a) and (b) diagrams depicted in Fig. 9 involving vector bosons $(W$ or $Z)$ and new charged $\left(\chi_{i=1,2}^{ \pm}\right)$or neutral $\left(\chi_{i=1 . .3}^{0}\right)$ fermions. It can be written as,

$$
i \delta Y=\sum_{j=(a),(b)}\left(i \delta Y_{j}^{\chi^{ \pm}}+i \delta Y_{j}^{\chi^{0}}\right),
$$

where

$$
\begin{aligned}
i \delta Y_{(a)}^{\chi^{ \pm}} & =-g^{2} \sum_{i, j=1}^{2}\left\{\left(O_{1 j}^{R} O_{1 i}^{L *} Y^{h \chi_{j}^{-} \chi_{i}^{+}}+O_{1 i}^{R} O_{1 j}^{L *} Y^{h \chi_{i}^{-} \chi_{j}^{+}}\right) I_{1}^{W i j}\right. \\
& +m_{\chi_{i}^{+}} m_{\chi_{j}^{+}}\left(O_{1 j}^{R} O_{1 i}^{L *} Y^{h \chi_{i}^{-} \chi_{j}^{+} *}+O_{1 i}^{R} O_{1 j}^{L *} Y^{h \chi_{j}^{-} \chi_{i}^{+} *}\right) I_{2}^{W i j} \\
& +\left[O_{1 j}^{L} O_{1 i}^{L *}\left(m_{\chi_{i}^{+}} Y^{h \chi_{i}^{-} \chi_{j}^{+} *}+m_{\chi_{j}^{+}} Y^{h \chi_{j}^{-} \chi_{i}^{+}}\right)\right. \\
& \left.\left.+O_{1 i}^{R} O_{1 j}^{R *}\left(m_{\chi_{i}^{+}} Y^{h \chi_{j}^{-} \chi_{i}^{+} *}+m_{\chi_{j}^{+}} Y^{h \chi_{i}^{-} \chi_{j}^{+}}\right)\right] I_{3}^{W i j}\right\}, \\
i \delta Y_{(a)}^{\chi^{0}} & =\frac{g^{2}}{c_{W}^{2}} \sum_{i, j=1}^{3}\left\{O_{j 1}^{\prime \prime L} O_{i 1}^{\prime \prime L} Y^{h \chi_{i}^{0} \chi_{j}^{0}} I_{1}^{Z i j}+m_{\chi_{i}^{0}} m_{\chi_{j}^{0}} O_{i 1}^{\prime \prime L} O_{j 1}^{\prime \prime L} Y^{h \chi_{i}^{0} \chi_{j}^{0} *} I_{2}^{Z i j}\right. \\
& \left.-O_{1 j}^{\prime \prime L} O_{i 1}^{\prime \prime L}\left(m_{\chi_{i}^{0}} Y^{h \chi_{i}^{0} \chi_{j}^{0} *}+m_{\chi_{j}^{0}} Y^{h \chi_{i}^{0} \chi_{j}^{0}}\right) I_{3}^{Z i j}\right\}, \\
i \delta Y_{(b)}^{\chi^{ \pm}}= & -\frac{\sqrt{2} g^{2} m_{W}^{2}}{v} \sum_{i=1}^{2}\left[\left(\left|O_{1 i}^{L}\right|^{2}+\left|O_{1 i}^{R}\right|^{2}\right) I_{4}^{W i}+2 m_{\chi_{i}^{+}} O_{1 i}^{L *} O_{1 i}^{R} I_{5}^{W i}\right], \\
i \delta Y_{(b)}^{\chi^{0}} & =-\frac{\sqrt{2} g^{2} m_{Z}^{2}}{c_{W}^{2} v} \sum_{i=1}^{3}\left\{O_{i 1}^{\prime \prime L} O_{1 i}^{\prime \prime L} I_{4}^{Z i}-m_{\chi_{i}^{0}}\left(O_{i 1}^{\prime \prime L}\right)^{2} I_{5}^{Z i}\right\}
\end{aligned}
$$


where the integrals, $I_{1 \ldots 5}^{V}$, are defined in terms of Passarino-Veltman (PV) functions [86] as,

$$
\begin{aligned}
& I_{1}^{V i j}=(D-1) m_{i}^{2} C_{0}\left(-p, p, m_{i}, m_{V}, m_{j}\right)-\frac{m_{i}^{2}}{m_{V}^{2}} B_{0}\left(0, m_{i}, m_{j}\right) \\
& +(D-1) B_{0}\left(p, m_{V}, m_{j}\right)-\frac{1}{m_{V}^{2}} A_{0}\left(m_{j}\right), \\
& I_{2}^{V i j}=(D-1) C_{0}\left(-p, p, m_{i}, m_{V}, m_{j}\right)-\frac{1}{m_{V}^{2}} B_{0}\left(0, m_{i}, m_{j}\right), \\
& I_{3}^{V i j}=\left(D-2+\frac{m_{i}^{2}}{m_{V}^{2}}-\frac{m_{\chi_{1}^{0}}^{2}}{m_{V}^{2}}\right) m_{\chi_{1}^{0}}\left[C_{11}\left(-p, p, m_{i}, m_{V}, m_{j}\right)-C_{12}\left(-p, p, m_{i}, m_{V}, m_{j}\right)\right] \\
& +\left(1+\frac{m_{i}^{2}}{m_{V}^{2}}-\frac{m_{\chi_{1}^{0}}^{2}}{m_{V}^{2}}\right) m_{\chi_{1}^{0}} C_{0}\left(-p, p, m_{i}, m_{V}, m_{j}\right)-\frac{m_{\chi_{1}^{0}}}{m_{V}^{2}} B_{1}\left(p, m_{V}, m_{j}\right) \\
& +\frac{m_{\chi_{1}^{0}}}{m_{V}^{2}} B_{0}\left(0, m_{i}, m_{j}\right) \text {, } \\
& I_{4}^{V i}=\left(2-D-\frac{m_{i}^{2}}{m_{V}^{2}}+\frac{m_{\chi_{1}^{0}}^{2}}{m_{V}^{2}}\right) m_{\chi_{1}^{0}}\left[C_{11}\left(p,-p, m_{V}, m_{i}, m_{V}\right)-C_{12}\left(p,-p, m_{V}, m_{i}, m_{V}\right)\right] \\
& \text { - }(D-3) m_{\chi_{1}^{0}} C_{0}\left(p,-p, m_{V}, m_{i}, m_{V}\right)+\frac{m_{\chi_{1}^{0}}}{m_{V}^{4}}\left(m_{i}^{2}-m_{\chi_{1}^{0}}^{2}\right) B_{1}\left(p, m_{V}, m_{i}\right) \\
& -\frac{m_{\chi_{1}^{0}}}{m_{V}^{4}} A_{0}\left(m_{i}\right) \\
& I_{5}^{V i}=(D-1) C_{0}\left(p,-p, m_{V}, m_{i}, m_{V}\right)+\frac{1}{m_{V}^{4}} A_{0}\left(m_{i}\right)
\end{aligned}
$$

where $D \equiv 4-2 \epsilon \delta_{\overline{\mathrm{MS}}}$ and $\delta_{\overline{\mathrm{MS}}}=1$ for $\overline{\mathrm{MS}}$ and $\delta_{\overline{\mathrm{MS}}}=0$ for $\overline{\mathrm{DR}}$ scheme. All external particles (i.e., $\chi_{1}^{0}$ ) are taken on-shell and $m_{i}=m_{\chi_{i}^{0}}$ for $V=Z$ and $m_{i}=m_{\chi_{i}^{ \pm}}$for $V=W$. Our notation for PV-functions $A, B, C$, follows closely the one defined in the Appendix of ref. [87. Functions $A_{0}, B_{0}, B_{1}$ contain both infinite and finite parts while $C_{0}, C_{11}, C_{12}$ - functions are purely finite. Our calculation has been done in unitary and (for a cross check) in Feynman gauge. The result for - $i \delta Y$ - is both renormalization scale invariant and finite.

\section{References}

[1] G. Bertone, D. Hooper, and J. Silk, "Particle dark matter: Evidence, candidates and constraints," Phys.Rept. 405 (2005) 279-390, arXiv:hep-ph/0404175 [hep-ph].

[2] XENON100 Collaboration, E. Aprile et al., "Dark Matter Results from 225 Live Days of XENON100 Data," Phys.Rev.Lett. 109 (2012) 181301, arXiv:1207.5988 [astro-ph.CO]. 
[3] LUX Collaboration, D. Akerib et al., "First results from the LUX dark matter experiment at the Sanford Underground Research Facility," arXiv:1310.8214 [astro-ph.CO].

[4] P. Panci, "New Directions in Direct Dark Matter Searches," arXiv:1402.1507 [hep-ph].

[5] M. W. Goodman and E. Witten, "Detectability of Certain Dark Matter Candidates," Phys.Rev. D31 (1985) 3059.

[6] Planck Collaboration, P. Ade et al., "Planck 2013 results. XVI. Cosmological parameters," arXiv:1303.5076 [astro-ph.CO].

[7] P. Sikivie, L. Susskind, M. B. Voloshin, and V. I. Zakharov, "Isospin Breaking in Technicolor Models," Nucl.Phys. B173 (1980) 189.

[8] F. del Aguila, A. Carmona, and J. Santiago, "Tau Custodian searches at the LHC," Phys.Lett. B695 (2011) 449-453, arXiv:1007.4206 [hep-ph].

[9] K. Agashe, R. Contino, L. Da Rold, and A. Pomarol, "A Custodial symmetry for Zb anti-b," Phys.Lett. B641 (2006) 62-66, arXiv:hep-ph/0605341 [hep-ph].

[10] R. Sekhar Chivukula, S. Di Chiara, R. Foadi, and E. H. Simmons, "The Limits of Custodial Symmetry," Phys.Rev. D80 (2009) 095001, arXiv:0908.1079 [hep-ph].

[11] A. Carmona and F. Goertz, "Custodial Leptons and Higgs Decays," arXiv:1301.5856 [hep-ph].

[12] H. E. Haber and G. L. Kane, "The Search for Supersymmetry: Probing Physics Beyond the Standard Model," Phys.Rept. 117 (1985) 75-263.

[13] M. S. Carena, A. Megevand, M. Quiros, and C. E. Wagner, "Electroweak baryogenesis and new TeV fermions," Nucl.Phys. B716 (2005) 319-351, arXiv:hep-ph/0410352 [hep-ph].

[14] R. Mahbubani and L. Senatore, "The Minimal model for dark matter and unification," Phys.Rev. D73 (2006) 043510, arXiv:hep-ph/0510064 [hep-ph].

[15] F. D'Eramo, "Dark matter and Higgs boson physics," Phys.Rev. D76 (2007) 083522 arXiv:0705.4493 [hep-ph].

[16] T. Cohen, J. Kearney, A. Pierce, and D. Tucker-Smith, "Singlet-Doublet Dark Matter," Phys.Rev. D85 (2012) 075003, arXiv:1109.2604 [hep-ph].

[17] M. Cirelli, N. Fornengo, and A. Strumia, "Minimal dark matter," Nucl.Phys. B753 (2006) 178-194, arXiv:hep-ph/0512090 [hep-ph].

[18] N. Arkani-Hamed and S. Dimopoulos, "Supersymmetric unification without low energy supersymmetry and signatures for fine-tuning at the LHC," JHEP 0506 (2005) 073. arXiv:hep-th/0405159 [hep-th].

[19] G. Giudice and A. Romanino, "Split supersymmetry," Nucl.Phys. B699 (2004) 65-89, arXiv:hep-ph/0406088 [hep-ph]. 
[20] CMS Collaboration, S. Chatrchyan et al., "Searches for long-lived charged particles in pp collisions at $\sqrt{s}=7$ and $8 \mathrm{TeV}$," arXiv:1305.0491 [hep-ex].

[21] XENON100 Collaboration, E. Aprile et al., "The XENON100 Dark Matter Experiment," Astropart.Phys. 35 (2012) 573-590, arXiv:1107.2155 [astro-ph.IM]

[22] C. Cheung, L. J. Hall, D. Pinner, and J. T. Ruderman, "Prospects and Blind Spots for Neutralino Dark Matter," arXiv:1211.4873 [hep-ph].

[23] C. Cheung and D. Sanford, "Simplified Models of Mixed Dark Matter," arXiv:1311.5896 [hep-ph].

[24] M. Drees, M. M. Nojiri, D. Roy, and Y. Yamada, "Light Higgsino dark matter," Phys.Rev. D56 (1997) 276-290, arXiv:hep-ph/9701219 [hep-ph].

[25] K. Griest and D. Seckel, "Three exceptions in the calculation of relic abundances," Phys.Rev. D43 (1991) 3191-3203.

[26] M. Pospelov and A. Ritz, "Higgs decays to dark matter: beyond the minimal model," Phys.Rev. D84 (2011) 113001, arXiv:1109.4872 [hep-ph].

[27] P. B. Pal, "Cold Dark Matter in the doublet - triplet Model," Phys.Lett. B205 (1988) 65.

[28] T. Araki, C. Geng, and K. I. Nagao, "Dark Matter in Inert Triplet Models," Phys.Rev. D83 (2011) 075014, arXiv:1102.4906 [hep-ph].

[29] S. S. Law and K. L. McDonald, "Inverse seesaw and dark matter in models with exotic lepton triplets," Phys.Lett. B713 (2012) 490-494, arXiv:1204.2529 [hep-ph].

[30] M. Aoki, J. Kubo, T. Okawa, and H. Takano, "Impact of Inert Higgsino Dark Matter," Phys.Lett. B707 (2012) 107-115, arXiv:1110.5403 [hep-ph].

[31] Y. G. Kim and K. Y. Lee, "The Minimal model of fermionic dark matter," Phys.Rev. D75 (2007) 115012, arXiv:hep-ph/0611069 [hep-ph].

[32] Y. G. Kim, K. Y. Lee, and S. Shin, "Singlet fermionic dark matter," JHEP 0805 (2008) 100, arXiv:0803.2932 [hep-ph].

[33] E. Ma and D. Suematsu, "Fermion Triplet Dark Matter and Radiative Neutrino Mass," Mod.Phys.Lett. A24 (2009) 583-589, arXiv:0809.0942 [hep-ph].

[34] K. Hamaguchi, S. Shirai, and T. Yanagida, "Cosmic Ray Positron and Electron Excess from Hidden-Fermion Dark Matter Decays," Phys.Lett. B673 (2009) 247-250, arXiv:0812.2374 [hep-ph].

[35] Y. G. Kim and S. Shin, "Singlet Fermionic Dark Matter explains DAMA signal," JHEP 0905 (2009) 036, arXiv:0901.2609 [hep-ph].

[36] C. de S. Pires, F. Queiroz, and P. Rodrigues da Silva, "Singlet Majorana fermion dark matter, DAMA, CoGeNT, and CDMS-II," Phys.Rev. D82 (2010) 105014, arXiv:1002.4601 [hep-ph]. 
[37] J.-M. Zheng, Z.-H. Yu, J.-W. Shao, X.-J. Bi, Z. Li, et al., "Constraining the interaction strength between dark matter and visible matter: I. fermionic dark matter," Nucl.Phys. B854 (2012) 350-374, arXiv: 1012.2022 [hep-ph].

[38] K. Fukushima, J. Kumar, and P. Sandick, "Detection Prospects for Majorana Fermion WIMPless Dark Matter," Phys.Rev. D84 (2011) 014020, arXiv:1103.5068 [hep-ph].

[39] B. Bellazzini, C. Csaki, J. Hubisz, J. Shao, and P. Tanedo, "Goldstone Fermion Dark Matter," JHEP 1109 (2011) 035, arXiv:1106.2162 [hep-ph].

[40] C.-H. Chen and S. S. Law, "Exotic fermion multiplets as a solution to baryon asymmetry, dark matter and neutrino masses," Phys.Rev. D85 (2012) 055012, arXiv:1111.5462 [hep-ph].

[41] E. Ma, "Dark-Matter Fermion from Left-Right Symmetry," Phys.Rev. D85 (2012) 091701, arXiv: 1202.5828 [hep-ph].

[42] W. Chao, "Dark Matter, LFV and Neutrino Magnetic Moment in the Radiative Seesaw Model with Triplet Fermion," arXiv:1202.6394 [hep-ph].

[43] L. Lopez-Honorez, T. Schwetz, and J. Zupan, "Higgs portal, fermionic dark matter, and a Standard Model like Higgs at 125 GeV," Phys.Lett. B716 (2012) 179-185, arXiv:1203.2064 [hep-ph].

[44] W.-M. Yang, "An Unification Model of Fermion Flavor and Baryon asymmetry and Dark Matter with The TeV Scale $U(1)_{B-L}$, arXiv:1206.5353 [hep-ph].

[45] H. Okada and T. Toma, "Fermionic Dark Matter in Radiative Inverse Seesaw Model with $U(1)_{B-L}$, " Phys.Rev. D86 (2012) 033011, arXiv:1207.0864 [hep-ph]

[46] S. Baek, P. Ko, W.-I. Park, and E. Senaha, "Vacuum structure and stability of a singlet fermion dark matter model with a singlet scalar messenger," arXiv:1209.4163 [hep-ph].

[47] H. K. Dreiner, H. E. Haber, and S. P. Martin, "Two-component spinor techniques and Feynman rules for quantum field theory and supersymmetry," Phys.Rept. 494 (2010) 1-196, arXiv:0812.1594 [hep-ph].

[48] J. Fan and M. Reece, "Probing Charged Matter Through Higgs Diphoton Decay, Gamma Ray Lines, and EDMs," JHEP 1306 (2013) 004, arXiv: 1301.2597.

[49] R. A. Horn and C. R. Johnson, "Matrix Analysis," Cambridge University Press (1995) p. 595.

[50] G. F. Giudice and A. Strumia, "Probing High-Scale and Split Supersymmetry with Higgs Mass Measurements," Nucl.Phys. B858 (2012) 63-83, arXiv:1108.6077 [hep-ph].

[51] N. Arkani-Hamed, K. Blum, R. T. D'Agnolo, and J. Fan, "2:1 for Naturalness at the LHC?," JHEP 1301 (2013) 149, arXiv:1207.4482 [hep-ph].

[52] G. Jungman, M. Kamionkowski, and K. Griest, "Supersymmetric dark matter," Phys.Rept. 267 (1996) 195-373, arXiv:hep-ph/9506380 [hep-ph]. 
[53] M. E. Peskin and T. Takeuchi, "Estimation of oblique electroweak corrections," Phys.Rev. D46 (1992) 381-409.

[54] Particle Data Group Collaboration, J. Beringer et al., "Review of Particle Physics (RPP)," Phys.Rev. D86 (2012) 010001.

[55] M. Drees and M. M. Nojiri, "The Neutralino relic density in minimal $N=1$ supergravity," Phys.Rev. D47 (1993) 376-408, arXiv:hep-ph/9207234 [hep-ph]

[56] M. Drees and M. Nojiri, "Neutralino - nucleon scattering revisited," Phys.Rev. D48 (1993) 3483-3501, arXiv:hep-ph/9307208 [hep-ph].

[57] A. Crivellin, M. Hoferichter, and M. Procura, "Accurate evaluation of hadronic uncertainties in spin-independent WIMP-nucleon scattering: Disentangling two- and three-flavor effects," Phys.Rev. D89 (2014) 054021, arXiv:1312.4951 [hep-ph].

[58] J. Gasser, H. Leutwyler, and M. Sainio, "Sigma term update," Phys.Lett. B253 (1991) 252-259.

[59] P. Junnarkar and A. Walker-Loud, "Scalar strange content of the nucleon from lattice QCD," Phys.Rev. D87 no. 11, (2013) 114510, arXiv:1301.1114 [hep-lat].

[60] J. R. Ellis, M. K. Gaillard, and D. V. Nanopoulos, "A Phenomenological Profile of the Higgs Boson," Nucl.Phys. B106 (1976) 292.

[61] A. Vainshtein, V. I. Zakharov, and M. A. Shifman, "Higgs Particles," Sov.Phys.Usp. 23 (1980) 429-449.

[62] B. A. Kniehl and M. Spira, "Low-energy theorems in Higgs physics," Z.Phys. C69 (1995) 77-88, arXiv:hep-ph/9505225 [hep-ph].

[63] A. Pilaftsis, "Higgs boson low-energy theorem and compatible gauge fixing conditions," Phys.Lett. B422 (1998) 201-211, arXiv:hep-ph/9711420 [hep-ph].

[64] J. Hisano, S. Matsumoto, M. M. Nojiri, and O. Saito, "Direct detection of the Wino and Higgsino-like neutralino dark matters at one-loop level," Phys.Rev. D71 (2005) 015007, arXiv:hep-ph/0407168 [hep-ph].

[65] J. Hisano, K. Ishiwata, N. Nagata, and T. Takesako, "Direct Detection of Electroweak-Interacting Dark Matter," JHEP 1107 (2011) 005, arXiv:1104.0228 [hep-ph].

[66] R. J. Hill and M. P. Solon, "WIMP-nucleon scattering with heavy WIMP effective theory," arXiv:1309.4092 [hep-ph].

[67] R. J. Hill and M. P. Solon, "Standard Model anatomy of WIMP dark matter direct detection I: weak-scale matching," arXiv:1401.3339 [hep-ph].

[68] J. Hisano, K. Ishiwata, and N. Nagata, "Direct Search of Dark Matter in High-Scale Supersymmetry," Phys.Rev. D87 (2013) 035020, arXiv:1210.5985 [hep-ph].

[69] T. Appelquist and J. Carazzone, "Infrared Singularities and Massive Fields," Phys.Rev. D11 (1975) 2856. 
[70] A. Dedes and K. Suxho, "Anatomy of the Higgs boson decay into two photons in the unitary gauge," Adv.High Energy Phys. 2013 (2013) 631841, arXiv:1210.0141 [hep-ph].

[71] A. Djouadi, "The Anatomy of electro-weak symmetry breaking. I: The Higgs boson in the standard model," Phys.Rept. 457 (2008) 1-216, arXiv:hep-ph/0503172 [hep-ph].

[72] A. Joglekar, P. Schwaller, and C. E. Wagner, "Dark Matter and Enhanced Higgs to Di-photon Rate from Vector-like Leptons," JHEP 1212 (2012) 064, arXiv:1207.4235 [hep-ph].

[73] CMS Collaboration, S. Chatrchyan et al., "Observation of a new boson at a mass of $125 \mathrm{GeV}$ with the CMS experiment at the LHC," Phys.Lett. B716 (2012) 30-61, arXiv:1207.7235 [hep-ex].

[74] ATLAS Collaboration, G. Aad et al., "Observation of a new particle in the search for the Standard Model Higgs boson with the ATLAS detector at the LHC," Phys.Lett. B716 (2012) 1-29, arXiv:1207.7214 [hep-ex].

[75] S. R. Coleman, "The Fate of the False Vacuum. 1. Semiclassical Theory," Phys.Rev. D15 (1977) 2929-2936.

[76] G. Isidori, G. Ridolfi, and A. Strumia, "On the metastability of the standard model vacuum," Nucl.Phys. B609 (2001) 387-409, arXiv:hep-ph/0104016 [hep-ph].

[77] C. Cheung, M. Papucci, and K. M. Zurek, "Higgs and Dark Matter Hints of an Oasis in the Desert," JHEP 1207 (2012) 105, arXiv:1203.5106 [hep-ph].

[78] ATLAS Collaboration, "Search for direct production of charginos and neutralinos in events with three leptons and missing transverse momentum in $21 \mathrm{fb}^{-1}$ of pp collisions at $\sqrt{s}=8 \mathrm{TeV}$ with the ATLAS detector," ATLAS-CONF-2013-035, ATLAS-COM-CONF-2013-042.

[79] CMS Collaboration, "Search for electroweak production of charginos, neutralinos, and sleptons using leptonic final states in pp collisions at $8 \mathrm{TeV}$," CMS-PAS-SUS-13-006.

[80] W. Beenakker, M. Klasen, M. Kramer, T. Plehn, M. Spira, et al., "The Production of charginos / neutralinos and sleptons at hadron colliders," Phys.Rev.Lett. 83 (1999) 3780-3783, arXiv:hep-ph/9906298 [hep-ph].

[81] J. F. Gunion and H. E. Haber, "Two-body decays of Neutralinos and Charginos," Phys.Rev. D37 (1988) 2515.

[82] A. Delgado, G. Nardini, and M. Quiros, "Large diphoton Higgs rates from supersymmetric triplets," Phys.Rev. D86 (2012) 115010, arXiv:1207.6596 [hep-ph].

[83] A. Freitas, S. Westhoff, and J. Zupan, "Integrating in the Higgs Portal to Fermion Dark Matter," arXiv:1506.04149 [hep-ph].

[84] T. Hahn, "Routines for the diagonalization of complex matrices," arXiv:physics/0607103 [physics]. 
[85] T. Hahn and M. Rauch, "News from FormCalc and LoopTools," Nucl.Phys.Proc.Suppl. 157 (2006) 236-240, arXiv:hep-ph/0601248 [hep-ph].

[86] G. Passarino and M. Veltman, "One Loop Corrections for $e^{+} e^{-}$Annihilation Into $\mu^{+} \mu^{-}$in the Weinberg Model," Nucl.Phys. B160 (1979) 151.

[87] A. Axelrod, "Flavor Changing $Z^{0}$ Decay and the Top Quark," Nucl.Phys. B209 (1982) 349 . 